\title{
Sense of Coherence in Nurses: A Systematic Review
}

\author{
Giuseppe Michele Masanotti ${ }^{1, *}$, Silvia Paolucci ${ }^{2}$, Elia Abbafati ${ }^{2}$, Claudio Serratore $^{2}$ and \\ Michela Caricato $^{2}$ \\ 1 Director of Experimental Centre of Research for Health Promotion and Health Education (CeSPES), \\ 06129 Perugia, Italy \\ 2 School of Specialization in Hygiene and Preventive Medicine, University of Perugia, 06129 Perugia, Italy; \\ silvia.paolucci01@gmail.com (S.P.); elia.abbafati@gmail.com (E.A.); claudio.serratore@gmail.com (C.S.); \\ michelacaricato@gmail.com (M.C.) \\ * Correspondence: giuseppe.masanotti@unipg.it; Tel.: +39-07-55-858-034
}

Received: 11 February 2020; Accepted: 8 March 2020; Published: 13 March 2020

\begin{abstract}
Background: Nurses experience high levels of distress due to the nature of their work and workplaces; Antonovsky's salutogenic theory shows that individual and work-related factors can influence human health. The aim of this paper is to analyze the possible correlations with different work-related and individual variables, which influence or are influenced by Sense of Coherence (SOC) and verify the possible use of SOC scales to prevent negative health determinants in workplaces. Methods: Electronic databases were searched with selected studies compared for sample, sample size, study design and basic results. Cross-sectional studies were reviewed for correlations between individual physical and mental health, distress, burnout, job satisfaction and SOC, with intervention studies used to assess the possible impact of training on nurses' SOC. Results: The review found several correlations between SOC and different work-related variables; but also with several individual characteristics. Conclusion: The review found that $\mathrm{SOC}$ was predictor of depressive state, burnout, job dissatisfaction among female nurses; therefore, SOC could be a health promoting resource.
\end{abstract}

Keywords: nurses; salutogenesis; Antonovsky

\section{Introduction}

Nurses are in the front line in the psychologically demanding everyday-care of patients, which can often undermine their emotional balance, influencing both their physical and mental wellbeing [1]. Moreover formal caregivers are frequently burdened with an excessive workload, high working pressure and demands, spending more time at work than on other dimensions of their lives [2,3]. All together these factors may contribute to the creation of a stressful working environment, which requires great coping abilities.

According to the salutogenic theory [4], one of the most critical determinants of the capacity to cope successfully with distress is the Sense of Coherence (SOC), which shapes the individual experience of a stressful event and allows it to be perceived as meaningful, manageable and comprehensible. This can be achieved by mobilizing efficiently the so-called GRRs (generalized resistance resources), which include biological, material and psychosocial factors, triggering a virtuous cycle and in turn strengthening the personal SOC [5]. Similarly, the Conservation of Resources theory (COR) states that the stress can result from circumstances involving loss of valued resources, and that the desire to preserve the individual resources is the basis of the coping ability [6].

Transposing Antonovsky's theory on the working context, the SOC can be modified, in a positive or negative way, by the nature of the current working environment. This re-adaptation explains how "job resources" are an integral part of the GRRs and participate in the modeling of the worker's SOC, which consequently influences how the so-called job demands (hours and pattern of work, workload, 
relations among the colleagues and every organizational aspects of a job that require continuous physical and/or psychological effort) are perceived, appraised, faced and overcome [5].

The SOC scale, named initially by Antonovsky's "Orientation to Life Questionnaire", investigating the three dimensions of SOC (Meaningfulness, Manageability and Comprehensibility) is available, to date, in two versions: the original form of 29 items (SOC-29) and the shorter version of 13 items (SOC-13). Each item is scored on a 7-point Likert scale, ranging 29-203 and 13-91, respectively, with higher score corresponding to a more developed SOC.

The aim of this paper is to analyze the possible correlations with different work-related and individual variables, which influence or are influenced by SOC and verify the possible use of SOC-29 or SOC-13 to prevent negative health determinants in workplaces.

\section{Materials and Methods}

\subsection{Research Methods}

A systematic search was conducted up to January 2019 on major healthcare databases: PubMed, Web of Science and Scopus. The following terms were included: sense of coherence, nurse, nurses, nursing, nursing staff, formal caregiver, formal caregivers; no additional filters used. Additional articles were retrieved from the consultation of relevant authors and paper's bibliography.

\subsection{Articles Selection}

Two independent reviewers selected the studies according to the following inclusion criteria: (1) original articles, and (2) administration of the SOC questionnaire to a sample of formal nurses.

Exclusion criteria applied were: (1) SOC questionnaire not administered, (2) language other than English, (3) sample different from working nurses (nurse teachers, unemployed nurses), (4) impossibility to retrieve a specific SOC value for the nurse sample, (5) absence of both mean SOC value and type of SOC questionnaire, and (6) use of other SOC questionnaire other than SOC-29 or SOC-13. Disagreements on article selection were resolved by consensus.

\subsection{Data Extraction and Synthesis}

Extraction of paper's data was independently performed by the reviewers through a pre-set table and consensus was reached, upon common revision, for each item inserted therein.

Selected papers were subsequently divided into three categories, based on whether the field of investigation of the Sense of Coherence was work-related or within the individual's sphere; articles assessing SOC variation upon interventions were categorized separately. The categories were named "Work-Related Variables", "Individual Variables" and "Interventions".

\section{Results}

A total of 876 papers were obtained. After duplicates removal, 535 records were screened initially by title and abstract and then by full text assessment. This process led to the exclusion of $n=454$ and $n=42$ articles respectively, yielding a total of 39 records included in the present review (Figure 1). 


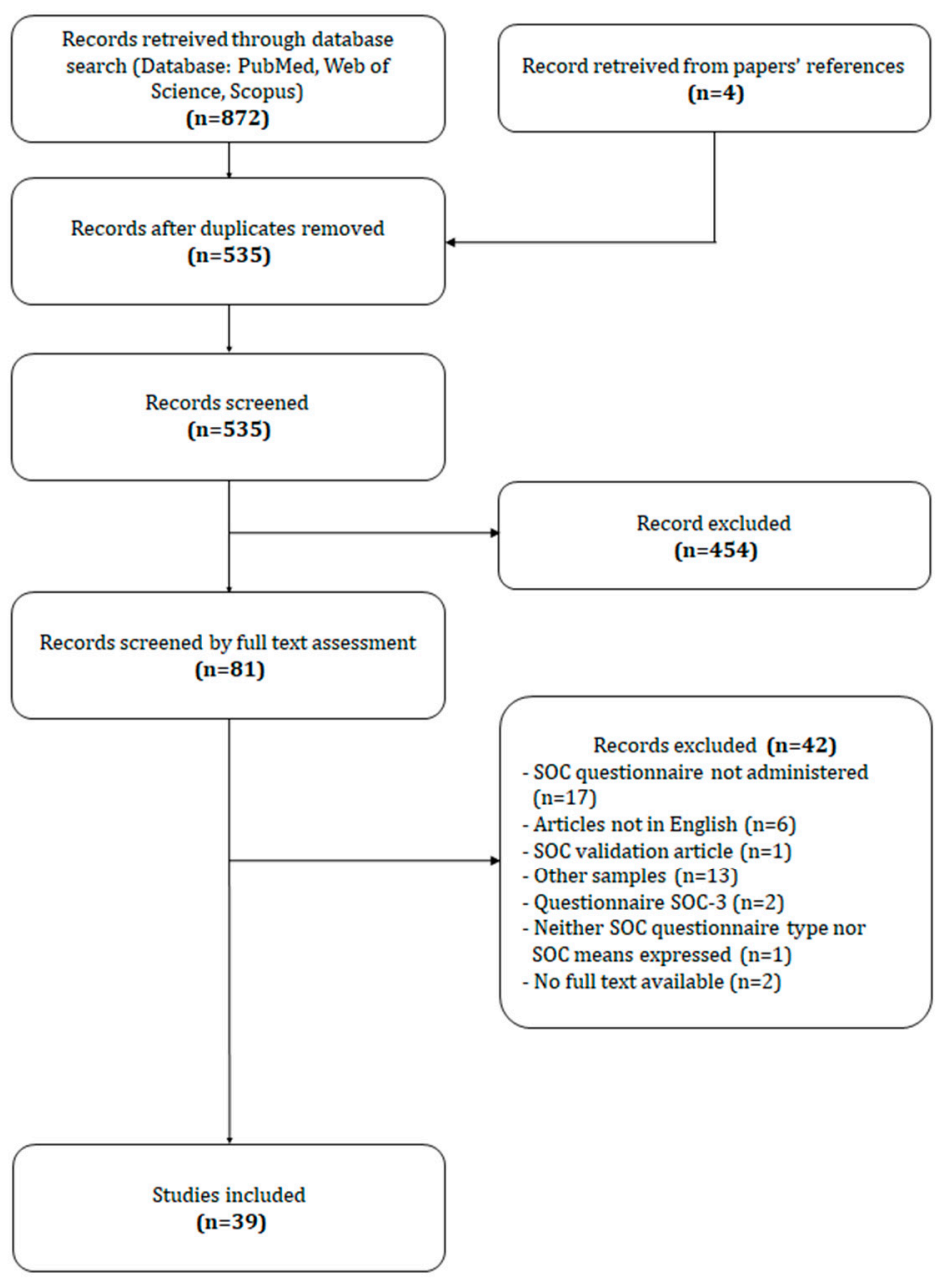

Figure 1. Flowchart of the searching and screening of literatures.

Data extraction from the included studies were performed and, according to the variables assessed, they were allocated to the three categories mentioned above: "Work-Related Variables", "Individual Variables", "Interventions" (Figure 2). Features of the same article, falling into more than one category were assessed separately. Table 1 summarizes the articles.

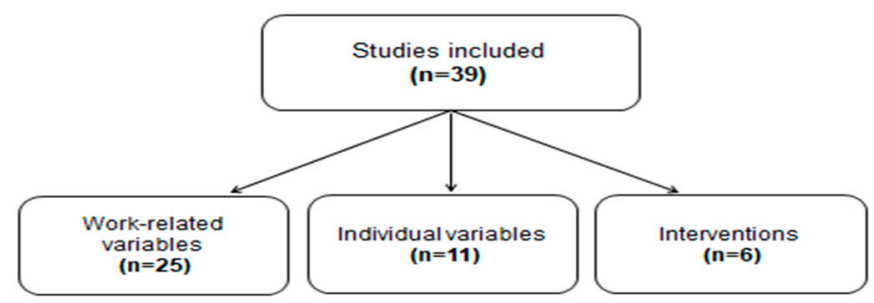

Figure 2. Flowchart of the allocation into the three categories: "Work-related Variables", "Individual Variables", "Interventions". 
Table 1. Included study.

\begin{tabular}{|c|c|c|c|c|c|c|c|c|c|c|}
\hline Author & Sample-Size/s & Sample & Country & $\begin{array}{l}\text { Mean Age } \\
\text { (SD) }\end{array}$ & $\begin{array}{c}\text { Mean } \\
\text { Experience } \\
\text { (SD) }\end{array}$ & SOC Scale & $\begin{array}{l}\text { Mean SOC } \\
\text { (SD) }\end{array}$ & Study Design & $\begin{array}{l}\text { Other Used } \\
\text { Instruments }\end{array}$ & $\begin{array}{l}\text { Sense of Coherence } \\
\text { (SOC)-Related Results }\end{array}$ \\
\hline $\begin{array}{l}\text { Kowitlawkul } \\
\text { et al., } \\
2018\end{array}$ & $\begin{array}{c}1040 \text { nurses } \\
955 \text { females } \\
(91.8 \%) \\
69 \text { males } \\
(6.6 \%) \\
16 \text { missing } \\
(1.5 \%)\end{array}$ & $\begin{array}{l}\text { Registered nurses and } \\
\text { enrolled nurses } \\
\text { working in inpatient } \\
\text { and outpatient } \\
\text { departments at a } \\
\text { tertiary hospital (in } \\
\text { the hospital for } \geq 6 \\
\text { months) }\end{array}$ & Singapore & $30.6(8.5)$ & - & SOC-13 & $\begin{array}{l}\text { 50/50 group: } \\
55.7 \text { (9.5) } \\
\text { 60/40 group: } \\
56.0 \text { (9.6) } \\
70 / 30 \text { group: } \\
54.5 \text { (9.2) } \\
\text { 80/20 group: } \\
52.4 \text { (10.8) }\end{array}$ & $\begin{array}{l}\text { Descriptive } \\
\text { quantitative study }\end{array}$ & $\begin{array}{c}\text { Work life balance } \\
\text { (WLB) } \\
\text { Job satisfaction } \\
\text { questionnaire } \\
\text { Social support } \\
\text { questionnaire } \\
\text { WHO-Quality of Life } \\
\text { (WHO-QOL)-BREF-26 } \\
\text { questionnaire }\end{array}$ & $\begin{array}{l}\text { - Division in four groups depending } \\
\text { on the proportion of time spent on } \\
\text { work and private life (50/50 and } \\
\text { below, } 60 / 40,70 / 30,80 / 20 \text { and above) } \\
\text {-“50/50 and below" and "60/40" } \\
\text { groups' SOC scores are higher the } \\
\text { other two groups-lowest SOC scores } \\
\text { are in the "80/20 and above" group } \\
\text {-SOC and social support are } \\
\text { significant predictors for all QoL } \\
\text { domains } \\
\text {-A unit increase in SOC results in a } \\
\text { 6-12\% increase in likelihood of } \\
\text { having high QoL for all domains }\end{array}$ \\
\hline $\begin{array}{l}\text { Kuraoka et al., } \\
2018\end{array}$ & $\begin{array}{c}63 \text { nurses } \\
60 \text { females } \\
(95.2 \%) \\
3 \text { males } \\
(4.8 \%)\end{array}$ & $\begin{array}{l}\text { Nurse managers in } \\
\text { the first } 3 \text { years of a } \\
\text { supervisory role, } \\
\text { working in acute-care } \\
\text { hospitals } \\
\text { Participation in an } \\
\text { experiential } \\
\text { learning-based } \\
\text { program }\end{array}$ & Japan & $45.2(3.4)$ & $1.4(0.6)$ & SOC-13 & $\begin{array}{l}\text { Pre-test: } \\
\text { 57.17 (10.28) } \\
\text { Post-test: } \\
54.97(10.4)\end{array}$ & $\begin{array}{l}\text { Quasi-experimental } \\
\text { study }\end{array}$ & $\begin{array}{c}\text { Experiential Learning } \\
\text { Inventory on the Job } \\
\text { (ELI) } \\
\text { Ad hoc questionnaire } \\
\text { for Knowledge of } \\
\text { Experiential Learning } \\
\text { Social Support } \\
\text { Questionnaire (SSQ) } \\
\text { Nurse Managers } \\
\text { Competence } \\
\text { Inventory (NMCI) }\end{array}$ & $\begin{array}{l}\text {-Mean SOC scores reduction after } \\
\text { participation to the experiential } \\
\text { learning-based program }\end{array}$ \\
\hline $\begin{array}{l}\text { Schäfer et al., } \\
2018\end{array}$ & $\begin{array}{c}27 \text { nurses } \\
44 \text { females } \\
(65.4 \%) \\
18 \text { males } \\
(34.6 \%)\end{array}$ & $\begin{array}{l}\text { Nurses working in } \\
\text { ICU and } \\
\text { anesthesiology unit }\end{array}$ & Germany & $39(10)$ & - & SOC-13 & 43.19 (9.63) & $\begin{array}{l}\text { Cross sectional } \\
\text { study }\end{array}$ & $\begin{array}{c}\text { Resilience Scale } \\
\text { (RS-11) } \\
\text { Scale for assessment } \\
\text { of internal and } \\
\text { external control } \\
\text { beliefs (IE-4) } \\
\text { ICD 10 symptom } \\
\text { rating (ISR) } \\
\text { PTSD Checklist } \\
\text { (PCL-5) }\end{array}$ & $\begin{array}{l}\text {-No differences in resilience, nor } \\
\text { SOC or LOC (locus of control) } \\
\text { between physicians and nursing } \\
\text { staff } \\
\text {-SOC, resilience, and internal and } \\
\text { external LOC correlate with ISR } \\
\text { Score and PTSD symptoms } \\
\text {-SOC is a significant predictor of } \\
\text { mental health problems and of } \\
\text { symptom severity } \\
\text {-Resilience, internal and external } \\
\text { LOC account for 59\% of SOC } \\
\text { variance and have a significant } \\
\text { indirect effects on symptom severity } \\
\text { measures mediated by SOC on } \\
\text { symptoms severity measures }\end{array}$ \\
\hline
\end{tabular}


Table 1. Cont

\begin{tabular}{|c|c|c|c|c|c|c|c|c|c|c|}
\hline Author & Sample-Size/sex & Sample & Country & $\begin{array}{l}\text { Mean Age } \\
\text { (SD) }\end{array}$ & $\begin{array}{c}\text { Mean } \\
\text { Experience } \\
\text { (SD) }\end{array}$ & SOC Scale & $\begin{array}{l}\text { Mean SOC } \\
\text { (SD) }\end{array}$ & Study Design & $\begin{array}{l}\text { Other Used } \\
\text { Instruments }\end{array}$ & $\begin{array}{l}\text { Sense of Coherence } \\
\text { (SOC)-Related Results }\end{array}$ \\
\hline $\begin{array}{l}\text { Debska et al., } \\
\quad 2017\end{array}$ & $\begin{array}{l}164 \text { nurses } \\
\text { (sex } \\
\text { unknown) }\end{array}$ & $\begin{array}{l}\text { Nurses working at } \\
\text { inpatient } \\
\text { chemotherapy wards }\end{array}$ & Poland & 43.07 (7.99) & $\begin{array}{l}\text { In } \\
\text { chemotherapy } \\
\text { ward: } 11.77 \\
(7.65)\end{array}$ & SOC-29 & $\begin{array}{l}125.05 \\
(18.30)\end{array}$ & $\begin{array}{l}\text { Cross-sectional } \\
\text { study }\end{array}$ & Meister Questionnaire & $\begin{array}{c}\text {-Highest SOC scores for the } \\
\text { Manageability subscale }(45.15), \\
\text { lower for Comprehensibility and } \\
\text { Meaningfulness subscales ( } 41.18 \\
\text { and } 38.73, \text { respectively) } \\
\text { - Inverse correlation between SOC } \\
\text { and Monotony }(\mathrm{r}=-0.398, p< \\
\text { 0.001), Unspecific Load }(\mathrm{r}=-0.370, \\
p<0.001),- \text { Mental Load }(\mathrm{r}= \\
-0.378, p<0.001) \text { and Work } \\
\text { experience }(\mathrm{r}=-0.19, p=0.016) \\
\text {-No correlation between SOC and } \\
\text { work-experience } \\
\text {-Inverse correlation between age } \\
\text { and Manageability level ( } \mathrm{r}=-0.193, \\
p=0.014) \\
\text {-No correlation between overall } \\
\text { SOC and age, nor educational level }\end{array}$ \\
\hline $\begin{array}{l}\text { Fusz et al., } \\
2017\end{array}$ & $\begin{array}{c}518 \text { nurses } \\
482 \text { females } \\
(93.1 \%) \\
36 \text { males } \\
(6.9 \%)\end{array}$ & $\begin{array}{l}\text { Nurses working in } \\
\text { eight different } \\
\text { hospitals }\end{array}$ & Hungary & $42.44(9.59)$ & - & SOC -13 & 61.76 & $\begin{array}{l}\text { Cross-sectional } \\
\text { study }\end{array}$ & $\begin{array}{l}\text { Ad hoc questionnaire } \\
\text { for sense of quality, } \\
\text { quality of sleep, } \\
\text { frequency of } \\
\text { psychosomatic } \\
\text { symptoms, and work } \\
\text { schedule regularity }\end{array}$ & $\begin{array}{c}\text {-Higher SOC score for day-shift } \\
\text { workers }(65.84), \text { compared to shift } \\
\text { workers }(61.02)(\mathrm{t}=2.933 ; p=0.004) \\
\text {-Lower SOC score for irregular work } \\
\text { schedule workers (58.19), compared } \\
\text { to flexible work schedule workers } \\
\text { (63.17, } p=0.04) \\
\text {-Lower scores in } \\
\text { self-heath-assessment for irregular } \\
\text { work schedule workers, compared } \\
\text { to flexible work schedule workers ( } \\
=0.019) \\
\end{array}$ \\
\hline $\begin{array}{l}\text { Stock et al., } \\
\quad 2017\end{array}$ & $\begin{array}{cc}12 \text { nurses } & \\
10 \text { females } & \mathrm{N} \\
(83.33 \%) & \text { ex } \\
2 \text { males } & \text { re } \\
(16.67 \%) & \end{array}$ & $\begin{array}{l}\text { Nurses self-described } \\
\text { as thriving, } \\
\text { experienced }(10+\text { y) or } \\
\text { recently retired }(<1 \text { y) }\end{array}$ & Hawaii & $\begin{array}{l}55-64 \mathrm{y}: \\
33.33 \%\end{array}$ & $\begin{array}{l}10-21 \text { y: } 33 \% \\
>34 \text { y: } 67 \%\end{array}$ & SOC-13 & 73.58 & $\begin{array}{l}\text { Mixed method, } \\
\text { exploratory-descrip } \\
\text { study }\end{array}$ & $\begin{array}{c}\text { Ad hoc questionnaire } \\
\text { vefor thriving view and } \\
\text { experience }\end{array}$ & $\begin{array}{c}\text { Possible link between SOC and } \\
\text { self-described thriving nurses } \\
\text { Nurses have a high SOC and the } \\
\text { use of GRRs }\end{array}$ \\
\hline
\end{tabular}


Table 1. Cont

\begin{tabular}{|c|c|c|c|c|c|c|c|c|c|c|}
\hline Author & Sample-Size/sex & Sample & Country & $\begin{array}{l}\text { Mean Age } \\
\text { (SD) }\end{array}$ & $\begin{array}{l}\text { Mean } \\
\text { Experience } \\
\text { (SD) }\end{array}$ & SOC Scale & $\begin{array}{l}\text { Mean SOC } \\
\text { (SD) }\end{array}$ & Study Design & $\begin{array}{l}\text { Other Used } \\
\text { Instruments }\end{array}$ & $\begin{array}{l}\text { Sense of Coherence } \\
\text { (SOC)-Related Results }\end{array}$ \\
\hline $\begin{array}{l}\text { Ando et al., } \\
\quad 2016\end{array}$ & $\begin{array}{c}130 \text { nurses } \\
102 \text { females } \\
(78.5 \%) \\
28 \text { males } \\
(21.5 \%)\end{array}$ & $\begin{array}{c}\text { Nurses from } \\
\text { psychiatric and } \\
\text { internal medicine } \\
\text { wards at a national } \\
\text { hospital }\end{array}$ & Japan & $\begin{array}{l}40-49 \text { y: } \\
35.4 \%\end{array}$ & 18.7 & SOC-13 & $53.5(9.7)$ & $\begin{array}{l}\text { Cross-sectional } \\
\text { study }\end{array}$ & $\begin{array}{c}\text { Moral Distress Scale } \\
\text { for Psychiatric Nurses } \\
\text { (MDS-P) } \\
\text { General Heath } \\
\text { Questionnaire - } 12 \\
\text { (GHQ-12) } \\
\text { Job Satisfaction Scale } \\
\text { (JS) }\end{array}$ & $\begin{array}{c}\text { Inverse correlation between MDS-P } \\
\text { and SOC } \\
\text { Inverse correlation between MDS-P } \\
\text { and JS } \\
\text { "Unethical conduct by caregivers" } \\
\text { negatively correlates with } \\
\text { Manageability }(\mathrm{r}=-0.28, p<0.01) \text {, } \\
\text { Comprehensibility }(\mathrm{r}=-0.22, p< \\
\text { 0.01) and Meaning }(\mathrm{r}=-0.017, \mathrm{p}< \\
0.05) \\
\text { "Low staffing" negatively correlated } \\
\text { with Comprehensibility }(\mathrm{r}=-0.30, \\
p<0.001) \text { and Manageability ( } \mathrm{r}= \\
-0.22, p<0.01) \\
\text { "Acquiescence to patients' rights } \\
\text { violations" negatively correlates } \\
\text { with Manageability ( } \mathrm{r}=-0.31, p< \\
0.01), \text { Comprehensibility }(\mathrm{r}=-0.28, \\
p<0.01) \text { and Meaning }(\mathrm{r}=-0.22, p \\
<0,01) . \\
\text { "Acquiescence to patients' rights } \\
\text { violations" (standard } \beta=-0,26, p< \\
0.01) \text { and "Meaning" of SOC } \\
\text { (standard } \beta=0.35, p<0.001) \\
\text { influence Job Satisfaction more than } \\
\text { other variables. }\end{array}$ \\
\hline $\begin{array}{c}\text { Lindmark et } \\
\text { al., } \\
2016\end{array}$ & $\begin{array}{l}165 \text { nurses } \\
\text { (sex } \\
\text { unknown) }\end{array}$ & $\begin{array}{l}\text { Dental nurses } \\
\text { working in the Public } \\
\text { Dental Service }\end{array}$ & Sweden & - & - & SOC-13 & $68.4(11.0)$ & $\begin{array}{l}\text { Cross-sectional } \\
\text { study }\end{array}$ & $\begin{array}{c}\text { Salutogenic Health } \\
\text { Indicator Scale (SHIS) } \\
\text { Work Experience } \\
\text { Measurement Scale } \\
\text { (WEMS) }\end{array}$ & $\begin{array}{c}\text { Clinical coordinators have higher } \\
\text { SOC score (75.1; SD 10.0), compared } \\
\text { with all other professions, including } \\
\text { dental nurses } \\
\text { Dental hygienists have higher } \\
\text { scores for meaningfulness (23.8; SD } \\
\text { 3.3), and dentists have higher scores } \\
\text { for manageability (22.1; SD 3.6), } \\
\text { compared with dental nurses (22.5; } \\
\text { SD 3.4 and 20.3; SD 4.0, } \\
\text { respectively) }\end{array}$ \\
\hline
\end{tabular}


Table 1. Cont.

\begin{tabular}{|c|c|c|c|c|c|c|c|c|c|c|}
\hline Author & Sample-Size/se & Sample & Country & $\begin{array}{l}\text { Mean Age } \\
\text { (SD) }\end{array}$ & $\begin{array}{l}\text { Mean } \\
\text { Experience } \\
\text { (SD) }\end{array}$ & SOC Scale & $\begin{array}{l}\text { Mean SOC } \\
\text { (SD) }\end{array}$ & Study Design & $\begin{array}{l}\text { Other Used } \\
\text { Instruments }\end{array}$ & $\begin{array}{l}\text { Sense of Coherence } \\
\text { (SOC)-Related Results }\end{array}$ \\
\hline $\begin{array}{l}\text { Vifladt et al., } \\
\quad 2016\end{array}$ & $\begin{array}{c}143 \text { nurses } \\
126 \text { females } \\
(88.1 \%) \\
13 \text { males } \\
(9.1 \%) \\
4 \text { unknown } \\
(2.8 \%)\end{array}$ & $\begin{array}{l}\text { Nurses working in } \\
\text { ICUs in six hospitals } \\
\text { for } \geq 3 \text { months }\end{array}$ & Norway & $\begin{array}{l}41-50 \mathrm{y}: \\
37.4 \%\end{array}$ & $\geq 21$ y: $26.1 \%$ & SOC-13 & $72.2(8.96)$ & $\begin{array}{l}\text { Cross-sectional } \\
\text { study }\end{array}$ & $\begin{array}{l}\text { Hospital Survey on } \\
\text { Patient Safety Culture } \\
\text { (HSOPSC) } \\
\text { Bergen Burnout } \\
\text { Indicator }\end{array}$ & $\begin{array}{c}\text { Positive safety culture negatively } \\
\text { correlates with burnout and } \\
\text { positively correlates with SOC }(\mathrm{r}= \\
-0.451, \mathrm{r}=0.393, \text { respectively, } p< \\
0.001) \text {. } \\
\text { Inverse correlation between SOC } \\
\text { and burnout }(\mathrm{r}=-0.577, p<0.001) \\
\text { Positive correlation between SOC } \\
\text { and safety culture at the unit level } \\
(\beta=0.014,95 \% \mathrm{CI}=0.005-0.024, p \\
0.003) \text { and hospital level }(\beta=0.017 \\
95 \% \mathrm{CI}=0.008-0.026, p<0.001)\end{array}$ \\
\hline $\begin{array}{l}\text { Yoshida et al., } \\
2016\end{array}$ & $\begin{array}{c}430 \text { nurses } \\
416 \text { females } \\
(96.7 \%) \\
14 \text { males } \\
(3.3 \%)\end{array}$ & $\begin{array}{l}\text { Public health nurses } \\
\text { (after the earthquake, } \\
\text { tsunami and } \\
\text { Fukushima Daiichi } \\
\text { Nuclear Power } \\
\text { Station accident } \\
\text { following the Great } \\
\text { East Japan } \\
\text { Earthquake in 2011) }\end{array}$ & Japan & $\begin{array}{l}\geq 50 \mathrm{y}: \\
35.6 \%\end{array}$ & $\begin{array}{l}<10 \text { y: } 22.8 \% \\
>10 \text { y: } 71.2 \%\end{array}$ & SOC-13 & $43.0(7.7)$ & $\begin{array}{l}\text { Cross-sectional } \\
\text { study }\end{array}$ & $\begin{array}{l}\text { Ad hoc questionnaire } \\
\text { for anxiety }\end{array}$ & $\begin{array}{r}\text { Division in two groups based on } \\
\text { anxiety score on a 10-point Likert } \\
\text { scale: 1-5 'anxiety }(-)^{\prime} \text { and } 6-10 \\
\text { 'anxiety }(+)^{\prime} \\
\text { No difference in mean SOC between } \\
\text { the anxiety }(+) \text { and the anxiety }(-) \\
\text { groups } \\
\text { Higher ratio of nurses }<40 \text { years of } \\
\text { age in the anxiety }(+) \text { group ( } p< \\
0.001) \\
\text { Higher ratio of nurses with }<10 \\
\text { years of working experience, staff } \\
\text { positions and nursing licenses in the } \\
\text { anxiety }(+) \text { group }(p<0.001)\end{array}$ \\
\hline $\begin{array}{l}\text { Hochwälder et } \\
\text { al., } \\
2015\end{array}$ & $\begin{array}{l}1012 \text { nurses } \\
\text { (all females) }\end{array}$ & $\begin{array}{l}\text { Nurses working at } \\
\text { three hospitals and } \\
\text { two primary health } \\
\text { care settings, } \geq 30 \mathrm{y} \text { of } \\
\text { age, with no negative } \\
\text { life events during the } \\
\text { previous year }\end{array}$ & Sweden & $46.6(8.9)$ & - & SOC-29 & $\begin{array}{c}150.21 \\
(20.28) \\
\text { Low SOC } \\
(<144) \text { group } \\
n=322): \\
126.35(13.82) \\
\text { Moderate } \\
\text { SOC } \\
(144-160) \\
\text { group } n= \\
348: 152.20 \\
(4.84) \\
\text { High SOC } \\
(>160) \text { group } \\
n=342: \\
170.63(7.49)\end{array}$ & Longitudinal study & $\begin{array}{l}\text { Ad hoc questionnaire } \\
\text { for Controllable and } \\
\text { Uncontrollable } \\
\text { Negative Life events }\end{array}$ & $\begin{array}{c}\text { No significant age differences } \\
\text { between Low, Moderate and High } \\
\text { SOC groups } \\
\text { Higher dropout rate in the Low } \\
\text { SOC group } \\
\text { No significant difference between } \\
\text { SOC groups and mean number of } \\
\text { uncontrolled negative life events } \\
\text { High and Moderate SOC groups } \\
\text { have fewer controllable negative life } \\
\text { events compared to the Low SOC } \\
\text { group }(p<0.001)\end{array}$ \\
\hline
\end{tabular}


Table 1. Cont

\begin{tabular}{|c|c|c|c|c|c|c|c|c|c|c|}
\hline Author & Sample-Size/se & Sample & Country & $\begin{array}{l}\text { Mean Age } \\
\text { (SD) }\end{array}$ & $\begin{array}{c}\text { Mean } \\
\text { Experience } \\
\text { (SD) }\end{array}$ & SOC Scale & $\begin{array}{l}\text { Mean SOC } \\
\text { (SD) }\end{array}$ & Study Design & $\begin{array}{l}\text { Other Used } \\
\text { Instruments }\end{array}$ & $\begin{array}{l}\text { Sense of Coherence } \\
\text { (SOC)-Related Results }\end{array}$ \\
\hline $\begin{array}{c}\text { Kretowicz et } \\
\text { al., } \\
2015\end{array}$ & $\begin{array}{c}310 \text { nurses } \\
301 \text { females } \\
(97.1 \%) \\
9 \text { males } \\
(2.9 \%)\end{array}$ & $\begin{array}{l}\text { Nurse managers in } \\
\text { selected medical units }\end{array}$ & Poland & $45.7(6.7)$ & $\begin{array}{c}\text { Mean } \\
\text { nursing } \\
\text { experience } \\
24.7(7.2) \text { y } \\
\text { Mean } \\
\text { managerial } \\
\text { experience } \\
8.8(6.7) \mathrm{y}\end{array}$ & SOC -29 & $\begin{array}{l}147.00 \\
(20.47)\end{array}$ & $\begin{array}{l}\text { Cross-sectional } \\
\text { study }\end{array}$ & - & $\begin{array}{c}\text { No correlation between age and } \\
\text { SOC } \\
\text { Correlation between educational } \\
\text { background and SOC } \\
\text { (Kruskal-Wallis test } 9.04 ; p=0.029 \text { ) } \\
\text { and Meaningfulness } \\
\text { (Kruskal-Wallis test } 12.82 ; p=0.005 \text { ) } \\
\text { No correlation between SOC and } \\
\text { general working experience, } \\
\text { managerial working experience, } \\
\text { position at work and characteristics } \\
\text { of employment } \\
\text { Higher SOC in nurses employed as } \\
\text { strategic managers (U Mann-Wallis } \\
\text { test }-2.74 ; p=0.006 \text { ) }\end{array}$ \\
\hline $\begin{array}{l}\text { Makabe et al., } \\
\quad 2015\end{array}$ & $\begin{array}{c}1202 \text { nurses } \\
1116 \text { females } \\
(93 \%) \\
86 \text { males } \\
(7 \%)\end{array}$ & $\begin{array}{l}\text { Nurses working in } \\
\text { three hospitals }\end{array}$ & Japan & $37(11)$ & $15(12)$ & SOC-13 & $\begin{array}{c}\text { 50/50 group } \\
\text { (A): } 55.7(9.5) \\
\text { 60/40 group } \\
\text { (B): } 56.0(9.6) \\
70 / 30 \text { group } \\
\text { (C): } 54.5(9.2) \\
80 / 20 \text { group } \\
\text { (D): } 52.4 \\
\text { (10.8) }\end{array}$ & $\begin{array}{l}\text { Cross sectional } \\
\text { study }\end{array}$ & $\begin{array}{c}\text { Work-Life Balance } \\
\text { (WLB) } \\
\text { Work-Life Balance } \\
\text { Satisfaction } \\
\text { Job Satisfaction Scale } \\
\text { WHO Quality of Life } \\
\text { (26-item) }\end{array}$ & $\begin{array}{c}\text { Division in four groups depending } \\
\text { on the proportions of percentages of } \\
\text { time spent on work and private life } \\
\text { WLB status [50/50 and below (A), } \\
60 / 40 \text { (B), } 70 / 30 \text { (C), } 80 / 20 \text { and above } \\
\text { (D)] } \\
\text { Group A has a higher SOC score } \\
\text { than all other groups (ANCOVA } p< \\
0.001 \text { ) } \\
\text { Group D has a lower SOC score } \\
\text { than all other groups (ANCOVA } p< \\
0.001 \text { ) }\end{array}$ \\
\hline $\begin{array}{l}\text { Miyata et al., } \\
2015\end{array}$ & $\begin{array}{c}1425 \text { nurses } \\
1333 \text { females } \\
(94 \%) \\
92 \text { males } \\
(6 \%)\end{array}$ & $\begin{array}{c}\text { Nurse staff }(n= \\
1248 \%-88 \%) \text { and } \\
\text { nurse managers }(n= \\
177-12 \%) \text { working in } \\
10 \text { hospitals }\end{array}$ & Japan & $35.5(9.9)$ & $12.8(9.5)$ & SOC-13 & $\begin{array}{l}\text { median: 50 } \\
\text { (IQR 45-55) }\end{array}$ & $\begin{array}{l}\text { Cross-sectional } \\
\text { study }\end{array}$ & $\begin{array}{l}\text { Recognition behavior } \\
\text { scale }\end{array}$ & $\begin{array}{c}\text { No significant correlation between } \\
\text { SOC and marital status } \\
\text { Positive correlation between SOC } \\
\text { and good mental health status (OR } \\
=4.07,95 \% \mathrm{CI}=2.53-6.53), \text { good } \\
\text { physical health status }(\mathrm{OR}=1.08, \\
95 \% \mathrm{CI}=1.09-2.89) \text {, overall work } \\
\text { experience }(\mathrm{OR}=1.05,95 \% \mathrm{CI}= \\
1.04-1.07) \text {, with } p<0.001 \text {, and } \\
\text { recognition behaviors by the nurse } \\
\text { manager (OR }=1.02(95 \% \mathrm{CI}= \\
1.01-1.04), p=0.006\end{array}$ \\
\hline
\end{tabular}


Table 1. Cont

\begin{tabular}{|c|c|c|c|c|c|c|c|c|c|c|}
\hline Author & Sample-Size/se & Sample & Country & $\begin{array}{l}\text { Mean Age } \\
\text { (SD) }\end{array}$ & $\begin{array}{c}\text { Mean } \\
\text { Experience } \\
\text { (SD) }\end{array}$ & SOC Scale & $\begin{array}{l}\text { Mean SOC } \\
\text { (SD) }\end{array}$ & Study Design & $\begin{array}{l}\text { Other Used } \\
\text { Instruments }\end{array}$ & $\begin{array}{l}\text { Sense of Coherence } \\
\text { (SOC)-Related Results }\end{array}$ \\
\hline $\begin{array}{l}\text { Kikuchi et al., } \\
2014\end{array}$ & $\begin{array}{c}347 \text { nurses } \\
\text { (all females) }\end{array}$ & $\begin{array}{l}\text { Nurses working at a } \\
\text { general hospital } \\
\text { (intensive care, } \\
\text { pediatrics, surgery, } \\
\text { oncology, and } \\
\text { emergency medicine) }\end{array}$ & Japan & $33.7(9.2)$ & - & SOC-13 & $54.2(11.9)$ & $\begin{array}{l}\text { Cross-sectional } \\
\text { study }\end{array}$ & $\begin{array}{c}\text { Brief Job Stress } \\
\text { Questionnaire (BJSQ) } \\
\text { K6 short screening } \\
\text { questionnaire } \\
\text { Ten-item Personality } \\
\text { Inventory (TIPI-J) }\end{array}$ & $\begin{array}{r}\text { Age negatively correlates with } \\
\text { depressive state }(\mathrm{r}=-0.18, p=0.00) \\
\text { and positively to SOC }(\mathrm{r}=0.27, p= \\
0.00) \\
\text { Inverse correlation between SOC } \\
\text { and shift work }(\mathrm{r}=-0.16, p=0.00), \\
\text { job rank }(\mathrm{r}=-0.14, p=0.01) \text {, and } \\
\text { overtime hours }(\mathrm{r}=-0.33, p=0.00) \\
\text { SOC and depressive state correlate } \\
\text { negatively with almost all job } \\
\text { stressors and personality traits } \\
\text { Inverse correlation between SOC } \\
\text { and Neuroticism }(\mathrm{r}=-0.49, p= \\
0.00) \text {. } \\
\text { Inverse correlation between SOC } \\
\text { and depressive state }(\mathrm{r}=-0.67, p= \\
0.00 \\
\text { Positive correlation between SOC } \\
\text { and job and life satisfaction }(\mathrm{r}= \\
0.47, p=0.00)\end{array}$ \\
\hline $\begin{array}{l}\text { Kikuchi et al., } \\
2014\end{array}$ & $\begin{array}{l}348 \text { nurses } \\
\text { (all females) }\end{array}$ & $\begin{array}{l}\text { Nurses working at a } \\
\text { general hospital } \\
\text { (intensive care, } \\
\text { pediatrics, surgery, } \\
\text { oncology, and } \\
\text { emergency medicine) }\end{array}$ & Japan & $34.4(9.0)$ & - & SOC-29 & $124.4(21.2)$ & $\begin{array}{l}\text { Cross-sectional } \\
\text { study }\end{array}$ & $\begin{array}{l}\text { K6 short screening } \\
\text { questionnaire } \\
\text { Effort-reward } \\
\text { imbalance (ERI) scale }\end{array}$ & $\begin{array}{c}\text { SOC }(\beta=-0.46, p<0.001) \\
\text { over-commitment, effort-esteem } \\
\text { ratio, and age significantly correlate } \\
\text { with the depressive state } \\
\text { Age correlates positively with SOC } \\
\quad(\mathrm{r}=0.12, p<0.05)\end{array}$ \\
\hline $\begin{array}{l}\text { Sarid et al., } \\
2012\end{array}$ & $\begin{array}{l}36 \text { nurses } \\
\text { (sex } \\
\text { unknown) }\end{array}$ & $\begin{array}{c}\text { Nurses working both } \\
\text { clinical and } \\
\text { administrative roles } \\
\text { in one major regional } \\
\text { hospital, } \geq 5 \text { y of } \\
\text { experience } \\
\text { Intervention group ( } n \\
=20 \text { ): Participation in } \\
\text { a } \\
\text { Cognitive-Behavioral } \\
\text { course } \\
\text { Control group }(n=16)\end{array}$ & Israel & $50.6(10.7)$ & - & SOC-13 & $\begin{array}{c}\text { Intervention } \\
\text { group } \\
\text { T1: } 70.83 \\
(7.67) \\
\text { T2: } 75.05 \\
(6.7) \\
\text { Control } \\
\text { group } \\
\text { T1: } 72.07 \\
(8.9) \\
\text { T2: } 69.61 \\
(7.64)\end{array}$ & $\begin{array}{l}\text { Pre-post test design, } \\
\text { with control }\end{array}$ & $\begin{array}{c}\text { Perceived stress scale } \\
\text { (PSS) } \\
\text { Profile of Mood states } \\
\text { (POMS) }\end{array}$ & $\begin{array}{l}\text { In the intervention group at T2 } \\
\text { higher SOC, more vigor, less } \\
\text { perceived stress, and less fatigue, } \\
\text { compared to T1 } \\
\text { At T1 no significant difference in } \\
\text { mean SOC scores between the } \\
\text { groups } \\
\text { At T2 significant difference in mean } \\
\text { SOC scores between the two groups } \\
\text { F(p) }=10.44(p<0.05)\end{array}$ \\
\hline
\end{tabular}


Table 1. Cont

\begin{tabular}{|c|c|c|c|c|c|c|c|c|c|c|}
\hline Author & Sample-Size/se & Sample & Country & $\begin{array}{l}\text { Mean Age } \\
\text { (SD) }\end{array}$ & $\begin{array}{l}\text { Mean } \\
\text { Experience } \\
\text { (SD) }\end{array}$ & SOC Scale & $\begin{array}{l}\text { Mean SOC } \\
\text { (SD) }\end{array}$ & Study Design & $\begin{array}{l}\text { Other Used } \\
\text { Instruments }\end{array}$ & $\begin{array}{l}\text { Sense of Coherence } \\
\text { (SOC)-Related Results }\end{array}$ \\
\hline $\begin{array}{l}\text { Ando et al., } \\
\quad 2011\end{array}$ & $\begin{array}{l}28 \text { nurses } \\
\text { (sex } \\
\text { unknown) }\end{array}$ & $\begin{array}{c}\text { Nurses working in } \\
\text { geriatric wards, } \geq 20 \mathrm{y} \\
\text { of experience, without } \\
\text { severe mental } \\
\text { problems } \\
\text { Intervention group ( } n \\
=15) \text { : participation in } \\
\text { mindfulness based } \\
\text { therapy sessions } \\
\text { Control Group ( } n= \\
\text { 13) }\end{array}$ & Japan & - & - & SOC-13 & $\begin{array}{l}\text { Post-interventic } \\
\text { Intervention } \\
\text { group: } 52 \\
\text { Control } \\
\text { group: } 53\end{array}$ & $\begin{array}{l}\text { Pre-post test design, } \\
\text { with control }\end{array}$ & $\begin{array}{c}\text { General Health } \\
\text { Questionnaire (GHQ) } \\
\text { Functional } \\
\text { Assessment of } \\
\text { Chronic Illness } \\
\text { Therapy (FACIT-sp) }\end{array}$ & $\begin{array}{c}\text { - Significant decrease in GHQ after } \\
\text { the intervention (improvement in } \\
\text { general health) } \\
\text { - Significant increase in SOC after } \\
\text { the intervention, no change in the } \\
\text { control group } \\
\text { - Meaningfulness: higher scores and } \\
\text { significant increase after } \\
\text { intervention: Higher scores and } \\
\text { significant increase after } \\
\text { intervention, compared to } \\
\text { comprehensibility and } \\
\text { manageability } \\
\text { - No effect on spirituality }\end{array}$ \\
\hline $\begin{array}{l}\text { Basinska et al., } \\
\quad 2011\end{array}$ & $\begin{array}{l}331 \text { nurses } \\
\text { (all females) }\end{array}$ & $\begin{array}{l}\text { Nurses working shifts } \\
\text { in three general care } \\
\text { hospitals }\end{array}$ & Poland & $34.15(6.61)$ & - & SOC-29 & $\begin{array}{l}136.46 \\
(21.43)\end{array}$ & $\begin{array}{c}\text { Descriptive } \\
\text { quantitative study }\end{array}$ & $\begin{array}{l}\text { Work Related } \\
\text { Patterns of Behavior } \\
\text { and Experience } \\
\text { Questionnaire } \\
\text { (AVEM) }\end{array}$ & $\begin{array}{c}\text { Division in work related behavior } \\
\text { type groups: type G-healthy, type } \\
\text { S-frugal, type A-risk } \\
\text { (overburdened), type B-burnout } \\
\text { Positive correlation between SOC } \\
\text { and healthy type } \mathrm{G} \text { and the frugal } \\
\text { type } \mathrm{S}(\mathrm{r}=0.50 \text {, and } \mathrm{r}=0.20 \\
\text { respectively, } p<0.001) \\
\text { Inverse correlation between SOC } \\
\text { and burnout type B and the } \\
\text { overburdened type } \mathrm{A}(\mathrm{r}=-0.57 p< \\
0.001 \text { and } \mathrm{r}=-0.13 p<0.05 \\
\text { respectively) } \\
\text { SOC explains } 28 \% \text { of the variability } \\
\text { of type B, } 21 \% \text { of type } \mathrm{G} \text { and } 7 \% \text { of } \\
\text { type S }\end{array}$ \\
\hline $\begin{array}{l}\text { Hochwälder et } \\
\text { al., } \\
2011\end{array}$ & $\begin{array}{l}1012 \text { nurses } \\
\text { (all females) }\end{array}$ & $\begin{array}{c}\text { Nurses working in } \\
\text { hospitals and primary } \\
\text { health care setting, > } \\
30 \text { y of age } \\
\text { Group 0: no negative } \\
\text { life events } \\
\text { Group 1: } \geq 1 \text { negative } \\
\text { life event } \\
\text { in the previous year }\end{array}$ & Sweden & $46.90(8.85)$ & $46.9(8.85)$ & SOC-29 & $\begin{array}{l}\text { At T1: } 150.21 \\
\quad(20.28) \\
\text { At T2: } 150.89 \\
\quad(20.38)\end{array}$ & $\begin{array}{l}\text { Cross sectional } \\
\text { study }\end{array}$ & $\begin{array}{l}\text { Ad hoc questionnaire } \\
\text { for Negative Life } \\
\text { Events }\end{array}$ & $\begin{array}{c}\text { SOC stable from T1 to T2 } \\
\text { No strong evidence that negative } \\
\text { life events lower SOC } \\
\text { No evidence that negative life } \\
\text { events lower SOC more in persons } \\
\text { with an initially low or moderate } \\
\text { SOC that in persons with an initially } \\
\text { high SOC. } \\
\text { SOC for those who experience a } \\
\text { negative life event, is initially } \\
\text { weaker, than those who do not } \\
\text { experience any negative life events }\end{array}$ \\
\hline
\end{tabular}


Table 1. Cont

\begin{tabular}{|c|c|c|c|c|c|c|c|c|c|c|}
\hline Author & Sample-Size/sex & Sample & Country & $\begin{array}{l}\text { Mean Age } \\
\text { (SD) }\end{array}$ & $\begin{array}{l}\text { Mean } \\
\text { Experience } \\
\text { (SD) }\end{array}$ & SOC Scale & $\begin{array}{l}\text { Mean SOC } \\
\text { (SD) }\end{array}$ & Study Design & $\begin{array}{l}\text { Other Used } \\
\text { Instruments }\end{array}$ & $\begin{array}{l}\text { Sense of Coherence } \\
\text { (SOC)-Related Results }\end{array}$ \\
\hline $\begin{array}{l}\text { Sarid et al., } \\
\quad 2010\end{array}$ & $\begin{array}{l}36 \text { nurses } \\
\text { (sex } \\
\text { unknow) }\end{array}$ & $\begin{array}{l}\text { Nurses working in all } \\
\text { hospital wards, }>5 \mathrm{y} \\
\text { of experience, both } \\
\text { clinical and } \\
\text { administrative roles } \\
\text { Study group }(n=20) \text { : } \\
\text { participation in a CBI } \\
\quad \text { course } \\
\text { Control group }(n=16)\end{array}$ & Israel & $50.6(10.7)$ & - & SOC-13 & - & Pre-post test design & $\begin{array}{l}\text { Perceived Stress Scale } \\
\text { (PSS) } \\
\text { Profile of Mood States } \\
\text { (POMS) }\end{array}$ & $\begin{array}{c}\text { No differences in SOC, perceived } \\
\text { stress and mood states between the } \\
\text { groups at T1 } \\
\text { Intervention group at T2: increased } \\
\text { levels of SOC and vigor and } \\
\text { decreased levels of perceived stress } \\
\text { and fatigue } \\
\text { Mean changes in SOC were }-4,20 \\
(\mathrm{SD}=1.28) \text { for the study group and } \\
2,11(\mathrm{SD}=1.46) \text { for the control } \\
\text { group, } \mathrm{F}=10.44, p<0.05\end{array}$ \\
\hline $\begin{array}{l}\text { Takeuchi et al., } \\
2010\end{array}$ & $\begin{array}{l}138 \text { nurses } \\
\text { (all females) }\end{array}$ & $\begin{array}{l}\text { Nurses working at } \\
\text { three hospitals, who } \\
\text { are also mothers } \\
\text { and/or wives }\end{array}$ & Japan & $36.2(8.0)$ & $12.5(8.3)$ & SOC-13 & $56.7(9.9)$ & $\begin{array}{c}\text { Descriptive } \\
\text { quantitative study }\end{array}$ & $\begin{array}{c}\text { Job Content } \\
\text { Questionnaire (JCQ, } 6 \\
\text { items) } \\
\text { Cumulative fatigue } \\
\text { symptoms index } \\
\text { (CFSI-18) } \\
\text { Center for } \\
\text { Epidemiologic } \\
\text { Studies Depression } \\
\text { (CES-D) scale } \\
\text { Work-to-Family } \\
\text { conflicts scale } \\
\end{array}$ & $\begin{array}{c}\text { Inverse correlation between } \\
\text { work-family conflict and SOC }(\beta= \\
-0.233, \text { partial } \mathrm{r}=-0.340, p<0.01) \\
\text { Inverse correlation between SOC } \\
\text { and cumulative fatigue }(\beta=-0.397 \text {, } \\
p<0.001) \text { and depression }(\beta= \\
\quad-0.517, p<0.001) . \\
\text { The interaction of SOC and WFC } \\
\text { influences depression }(\beta=0.214, p \\
<0.05), \text { SOC has a buffering effect } \\
\text { on WFC with respect to depression. }\end{array}$ \\
\hline $\begin{array}{l}\text { Ida et al., } \\
2009\end{array}$ & $\begin{array}{l}502 \text { nurses } \\
\text { (all females) }\end{array}$ & $\begin{array}{l}\text { Nurses working at a } \\
\text { major university } \\
\text { hospital }\end{array}$ & Japan & $32.4(9.9)$ & - & SOC-29 & - & $\begin{array}{l}\text { Cross-sectional } \\
\text { study }\end{array}$ & $\begin{array}{c}\text { Job Content } \\
\text { Questionnaire } \\
\text { (JCQ-12) } \\
\text { Ad hoc questionnaire } \\
\text { for medical errors and } \\
\text { nurses career levels }\end{array}$ & $\begin{array}{c}\text { Positive correlation between } \\
\text { Comprehensibility and professional } \\
\text { experience }(\mathrm{r}=0.125, p<0.001) \\
\text { Positive correlation between SOC } \\
\text { and workplace adaptability }(\mathrm{r}= \\
0.335, p<0.01), \text { and job satisfaction } \\
(\mathrm{r}=0.280, p<0.01) \\
\text { Inverse correlation between SOC } \\
\text { and organization environment }(\mathrm{r}= \\
-0.611, p<0.01) \text { and health risk }(\mathrm{r}= \\
-0.364, p<0.01) \\
\text { SOC affects sickness-absence }(\mathrm{OR}= \\
0.982,95 \% \mathrm{CI}=0.970-0.995)\end{array}$ \\
\hline
\end{tabular}


Table 1. Cont

\begin{tabular}{|c|c|c|c|c|c|c|c|c|c|c|}
\hline Author & Sample-Size/sex & Sample & Country & $\begin{array}{l}\text { Mean Age } \\
\text { (SD) }\end{array}$ & $\begin{array}{c}\text { Mean } \\
\text { Experience } \\
\text { (SD) }\end{array}$ & SOC Scale & $\begin{array}{l}\text { Mean SOC } \\
\text { (SD) }\end{array}$ & Study Design & $\begin{array}{l}\text { Other Used } \\
\text { Instruments }\end{array}$ & $\begin{array}{l}\text { Sense of Coherence } \\
\text { (SOC)-Related Results }\end{array}$ \\
\hline $\begin{array}{l}\text { Van der Colff } \\
\text { et al., } \\
2009\end{array}$ & $\begin{array}{c}818 \text { nurses } \\
791 \text { females } \\
(97.4 \%) \\
21 \text { males } \\
(2.6 \%) \\
6 \text { sex } \\
\text { unknown }\end{array}$ & $\begin{array}{l}\text { Nurses working in } \\
\text { hospital wards, } \\
\text { psychiatric wards, } \\
\text { community/occupational } \\
\text { services and nursing } \\
\text { management. }\end{array}$ & South Africa & 40 & 19 & SOC-29 & $\begin{array}{l}137.95 \\
(20.46)\end{array}$ & $\begin{array}{l}\text { Cross sectional } \\
\text { study }\end{array}$ & $\begin{array}{c}\text { Nursing Stress } \\
\text { Inventory (NSI) } \\
\text { Coping Orientation } \\
\text { for Problem } \\
\text { Experienced } \\
\text { Questionnaire } \\
\text { (COPE) } \\
\text { Maslach Burnout } \\
\text { Inventory- } \\
\text { Human Services } \\
\text { Survey (MBIHSS) } \\
\text { Utrecht Work } \\
\text { Engagement Scale } \\
\text { (UWES) }\end{array}$ & $\begin{array}{c}\text { Correlation between SOC and } \\
\text { burnout subscores. Emotional } \\
\text { exhaustion }(-0,49), \\
\text { Depersonalization }(-0,47) \text { and } \\
\text { Personal accomplishment }(0.34)(p< \\
0.05) \\
\text { Positive correlation between SOC } \\
\text { and Engagement }(0.42) \\
\text { Negative correlation between SOC } \\
\text { and Nursing stress inventory (NSI) } \\
\text { variables: Lack of organizational } \\
\text { support }(-0.23) \text {, job demands } \\
(-0.28) \text { and nursing-specific } \\
\text { demands }(-0.15)(p<0.05) \\
\text { SOC correlates with coping } \\
\text { strategies: Approach coping }(0.0), \\
\text { Seeking emotional/social support } \\
(0.18) \text { and Turning to religion }(0,11) \\
\text { Avoidance }(-0.35) \text { and Focus on } \\
\text { and ventilation of emotions }(-0.29) \\
(p<0.05)\end{array}$ \\
\hline $\begin{array}{l}\text { Hall-Lord et } \\
\text { al., } \\
2006\end{array}$ & $\begin{array}{c}71 \text { nurses } \\
\text { (sex not } \\
\text { specified) }\end{array}$ & $\begin{array}{l}\text { Nurses participating } \\
\text { in an educational } \\
\text { program }\end{array}$ & Sweden & $39.2(8.05)$ & $16.7(7.35)$ & SOC-13 & - & $\begin{array}{l}\text { Cross-sectional } \\
\text { study }\end{array}$ & $\begin{array}{l}\text { Ad hoc questionnaire } \\
\text { for assessment of pain } \\
\text { and distress of } \\
\text { patients } \\
\text { Five-factor } \\
\text { personality inventory } \\
\text { (FFPI) }\end{array}$ & $\begin{array}{l}\text { Patients' age and type of illness } \\
\text { seems to influence nurses' } \\
\text { assessments of pain and distress } \\
\text { Nurses with high emotional } \\
\text { stability and high SOC scores assess } \\
\text { pain and distress for acute patients } \\
\text { as less intense and assess it more } \\
\text { intense for chronic patients }\end{array}$ \\
\hline
\end{tabular}


Table 1. Cont

\begin{tabular}{|c|c|c|c|c|c|c|c|c|c|c|}
\hline Author & Sample-Size/se & Sample & Country & $\begin{array}{l}\text { Mean Age } \\
\text { (SD) }\end{array}$ & $\begin{array}{c}\text { Mean } \\
\text { Experience } \\
\text { (SD) }\end{array}$ & SOC Scale & $\begin{array}{l}\text { Mean SOC } \\
\text { (SD) }\end{array}$ & Study Design & $\begin{array}{l}\text { Other Used } \\
\text { Instruments }\end{array}$ & $\begin{array}{l}\text { Sense of Coherence } \\
\text { (SOC)-Related Results }\end{array}$ \\
\hline $\begin{array}{c}\text { Engström et } \\
\text { al., } \\
2005\end{array}$ & $\begin{array}{c}33 \text { nurses } \\
31 \text { females } \\
(94 \%) \\
2 \text { males }(6 \%)\end{array}$ & $\begin{array}{l}\text { Nurses working in a } \\
\text { residential home for } \\
\text { persons with } \\
\text { dementia } \\
\text { Experimental group } \\
(n=17) \text { : participation } \\
\text { in IT support project } \\
\text { Control group }(n=16)\end{array}$ & Sweden & 41 & $\begin{array}{l}\text { Experimental } \\
\text { group } \\
\text {-in nursing } \\
\text { care } 12(8) \\
\text {-in dementia } \\
\text { care } 5(3) \\
\text { Control } \\
\text { group } \\
\text {-in nursing } \\
\text { care } 15(9) \\
\text {-as district } \\
\text { nurse } 9(7)\end{array}$ & SOC-13 & $\begin{array}{c}\text { Intervention } \\
\text { group } \\
\text {-baseline: } 72 \\
\text { (12) } \\
\text {-at } 6 \text { months: } \\
69(11) \\
\text {-at } 12 \\
\text { months: } 75 \\
\text { (9) } \\
\text { Control } \\
\text { group } \\
\text {-baseline: } 68 \\
\text { (10) } \\
\text {-at } 6 \text { months: } \\
69(9) \\
\text {-at 12 } \\
\text { months: } 65 \\
\text { (13) }\end{array}$ & $\begin{array}{l}\text { Quasi-experimental } \\
\text { non-equivalent } \\
\text { groups design }\end{array}$ & $\begin{array}{c}\text { Satisfaction with } \\
\text { Work Questionaires } \\
\text { (SWQ) } \\
\text { Life Satisfaction } \\
\text { Questionnaire (LSQ) }\end{array}$ & $\begin{array}{l}\text { Perception of psychosocial job } \\
\text { satisfaction and quality of care } \\
\text { improve in the experimental group } \\
\text { No significant within-subject effect } \\
\text { for the total SOC scale and } \\
\text { meaningfulness subscale, split on } \\
\text { the experimental and control groups } \\
\text { Interaction effect for the factors } \\
\text { family relation, close friend relation } \\
\text { (LSQ), total SOC scale and } \\
\text { meaningfulness subscale }\end{array}$ \\
\hline $\begin{array}{l}\text { Höge et al., } \\
2004\end{array}$ & $\begin{array}{c}160 \text { nurses } \\
\text { (sex not } \\
\text { specified) }\end{array}$ & $\begin{array}{l}\text { Nurses working at } \\
\text { two hospitals }\end{array}$ & Germany & - & - & SOC-13 & $68.1(10.83)$ & $\begin{array}{l}\text { Cross-sectional } \\
\text { study }\end{array}$ & $\begin{array}{c}\text { Work load screening } \\
\text { TAA-KH-S } \\
\text { Negative Affectivity } \\
\text { Scale (NAS) } \\
\text { Maslach Burnout } \\
\text { Inventory (Emotional } \\
\text { exhaustion } \\
\text { subcategory) } \\
\text { Irritation Strain } \\
\text { questionnaire } \\
\text { Short Form Health } \\
\text { Survey (SF-12) }\end{array}$ & $\begin{array}{c}\text { Inverse correlation between SOC } \\
\text { and Negative Affectivity }(\mathrm{r}=-0.61) \\
\text { Inverse correlation between SOC } \\
\text { and overall strain }(\mathrm{rs}=-0.33) \text { and } \\
\text { overall stressors }(\mathrm{rs}=-0.34)\end{array}$ \\
\hline $\begin{array}{l}\text { Shimizu et al., } \\
\quad 2004\end{array}$ & $\begin{array}{l}285 \text { nurses } \\
\text { (all females) }\end{array}$ & $\begin{array}{l}\text { Nurses working at a } \\
\quad \text { hospital } \\
\text { Intervention group ( } n \\
=62 \text { ): participation in } \\
\text { an assertiveness } \\
\text { training } \\
\text { Reference group ( } n= \\
\text { 196) }\end{array}$ & Japan & $\begin{array}{c}\text { Intervention } \\
\text { group: } 44 \\
\text { (7.1) } \\
\text { Reference } \\
\text { group: } 27.8 \\
\quad(5.9)\end{array}$ & - & SOC-13 & $\begin{array}{c}\text { Intervention } \\
\text { group } \\
\text { T1: } 57.4 \\
(10.5) \\
\text { T2: } \Delta \text { SOC } \\
2.2(8.3) \\
\text { Comparison } \\
\text { group } \\
\text { T1: } 52.7(9.4) \\
\text { T2: } \Delta \text { SOC } \\
1.5(8.6)\end{array}$ & $\begin{array}{l}\text { Pre-post test design, } \\
\text { with control }\end{array}$ & $\begin{array}{c}\text { Rosemberg's } \\
\text { self-esteem scale (SES) }\end{array}$ & $\begin{array}{l}\text {-No significant difference between } \\
\text { the } \triangle \text { SOC of the intervention group } \\
\text { and that of the reference group. } \\
\text {-Improvement in SES at six months } \\
\text { after the intervention }\end{array}$ \\
\hline
\end{tabular}


Table 1. Cont

\begin{tabular}{|c|c|c|c|c|c|c|c|c|c|c|}
\hline Author & Sample-Size/sex & Sample & Country & $\begin{array}{l}\text { Mean Age } \\
\text { (SD) }\end{array}$ & $\begin{array}{l}\text { Mean } \\
\text { Experience } \\
\text { (SD) }\end{array}$ & SOC Scale & $\begin{array}{l}\text { Mean SOC } \\
\text { (SD) }\end{array}$ & Study Design & $\begin{array}{l}\text { Other Used } \\
\text { Instruments }\end{array}$ & $\begin{array}{l}\text { Sense of Coherence } \\
\text { (SOC)-Related Results }\end{array}$ \\
\hline $\begin{array}{l}\text { Cilliers et al., } \\
\quad 2003\end{array}$ & $\begin{array}{l}105 \text { nurses } \\
\text { (all females) }\end{array}$ & $\begin{array}{l}\text { Nurses working in } \\
\text { large hospitals, } \geq 5 \mathrm{y} \\
\text { of experience }\end{array}$ & South Africa & range: $28-57$ & & SOC-29 & $\begin{array}{l}141.28 \\
(16.44)\end{array}$ & $\begin{array}{l}\text { Cross-sectional } \\
\text { study }\end{array}$ & $\begin{array}{c}\text { Maslach Burnout } \\
\text { Inventory (MBI) } \\
\text { Personal Views } \\
\text { Survey (HAR) } \\
\text { Self-Control Schedule } \\
\text { (LR) }\end{array}$ & $\begin{array}{c}\text { Inverse correlation between } \\
\text { burnout and salutogenic } \\
\text { functioning (SOC, HAR and LR) } \\
\text { Inverse correlation between SOC } \\
\text { and emotional exhaustion }(\mathrm{r}= \\
-0.21, p<0.01) \text { and } \\
\text { depersonalization }(\mathrm{r}=-0.25, p< \\
0.01) \\
\text { Positive correlation between SOC } \\
\text { and personal accomplishment }(\mathrm{r}= \\
0.35, p<0.001)\end{array}$ \\
\hline $\begin{array}{l}\text { Yam et al., } \\
2003\end{array}$ & $\begin{array}{l}29 \text { nurses } \\
28 \text { females } \\
(96.5 \%) \\
1 \text { male } \\
(3.4 \%)\end{array}$ & $\begin{array}{l}\text { Nurses working in } \\
\text { critical care in public } \\
\text { and private hospitals }\end{array}$ & Hong Kong & 32 & 7.6 & SOC-13 & - & $\begin{array}{l}\text { Cross-sectional } \\
\text { study }\end{array}$ & $\begin{array}{c}\text { Critical Care Nursing } \\
\text { stress scale (CCNSS) } \\
\text { Perceived Stress Scale } \\
\text { (PSS) }\end{array}$ & $\begin{array}{l}\text { Inverse correlation between SOC } \\
\text { and CCNSS }(\mathrm{r}=-0.20, p=0.30) \\
\text { Inverse correlation between SOC } \\
\text { and PSS }(\mathrm{r}=-0.64, p<0.001)\end{array}$ \\
\hline $\begin{array}{l}\text { Michael et al., } \\
2001\end{array}$ & $\begin{array}{c}233 \text { nurses } \\
225 \text { females } \\
(96.56 \%) \\
8 \text { males } \\
(3.43 \%)\end{array}$ & $\begin{array}{l}\text { Nurses working in } \\
\text { operating suites in } \\
\text { private and public } \\
\text { hospitals }\end{array}$ & Australia & 41 & $\begin{array}{l}11 \text { years in } \\
\text { operating } \\
\text { suite }\end{array}$ & SOC-13 & $66.75(9.77)$ & $\begin{array}{l}\text { Mixed method } \\
\text { trianglulated study }\end{array}$ & $\begin{array}{l}\text { Ad hoc questionnaire } \\
\text { for work-related } \\
\text { traumatic events } \\
\text { Ad hoc questionnaire } \\
\text { for social support }\end{array}$ & $\begin{array}{l}\text { nurses who did not experience a } \\
\text { traumatic event had a higher SOC } \\
(\mathrm{t}(231)=-3.12, p<0.005)\end{array}$ \\
\hline $\begin{array}{l}\text { Tselebis et al., } \\
\quad 2001\end{array}$ & $\begin{array}{l}79 \text { nurses } \\
62 \text { females } \\
(78.5 \%) \\
17 \text { males } \\
(21.5 \%)\end{array}$ & $\begin{array}{l}\text { Nurses working in } \\
\text { general internal } \\
\text { medicine, general } \\
\text { surgery and } \\
\text { respiratory medical } \\
\text { wards in a major } \\
\text { hospital }\end{array}$ & Greece & $37.7(5.5)$ & $11.0(6.3)$ & SOC-13 & $63.60(11.70)$ & $\begin{array}{l}\text { Cross-sectional } \\
\text { study }\end{array}$ & $\begin{array}{l}\text { Maslach Burnout } \\
\text { Inventory (MBI) } \\
\text { Beck's Depression } \\
\text { Inventory (BDI) }\end{array}$ & $\begin{array}{c}\text { No differences in SOC between } \\
\text { sexes nor marital status } \\
\text { Inverse correlation between SOC } \\
\text { and BDI }(\mathrm{r}=-0.58, p<0.05) \\
\text { Correlation between SOC and MBI } \\
\text { categories, negative correlation with } \\
\text { sentimental exhaustion }(\mathrm{r}=-0.55, p \\
<0.05) \text { and depersonalization }(\mathrm{r}= \\
-0.45, p<0.05) \text {, positive correlation } \\
\text { with personal achievement }(\mathrm{r}=0.44 \\
\quad p<0.05)\end{array}$ \\
\hline
\end{tabular}


Table 1. Cont

\begin{tabular}{|c|c|c|c|c|c|c|c|c|c|c|}
\hline Author & Sample-Size/sex & Sample & Country & $\begin{array}{l}\text { Mean Age } \\
\text { (SD) }\end{array}$ & $\begin{array}{c}\text { Mean } \\
\text { Experience } \\
\text { (SD) }\end{array}$ & SOC Scale & $\begin{array}{l}\text { Mean SOC } \\
\text { (SD) }\end{array}$ & Study Design & $\begin{array}{l}\text { Other Used } \\
\text { Instruments }\end{array}$ & $\begin{array}{l}\text { Sense of Coherence } \\
\text { (SOC)-Related Results }\end{array}$ \\
\hline $\begin{array}{l}\text { Levert et al., } \\
2000\end{array}$ & $\begin{array}{c}94 \text { nurses } \\
67 \text { females } \\
(71.3 \%) \\
27 \text { male } \\
(28.7 \%)\end{array}$ & $\begin{array}{l}\text { Nurses working in } \\
\text { psychiatric units }\end{array}$ & South Africa & 39 & - & SOC-13 & $60.61(12.42)$ & $\begin{array}{l}\text { Cross-sectional } \\
\text { study }\end{array}$ & $\begin{array}{l}\text { Maslach Burnout } \\
\text { Inventory (MBI) } \\
\text { Work Load and Lack } \\
\text { of Collegial Support. } \\
\text { Role Conflict and } \\
\text { Role Ambiguity }\end{array}$ & 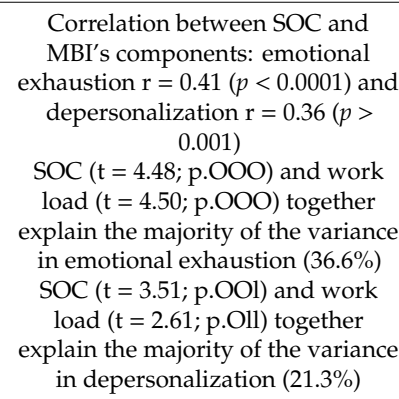 \\
\hline $\begin{array}{l}\text { Berg et al., } \\
\quad 1999\end{array}$ & $\begin{array}{c}22 \text { nurses } \\
16 \text { females } \\
(72.7 \%) \\
6 \text { male } \\
(27.3 \%)\end{array}$ & $\begin{array}{l}\text { Nurses working in } \\
\text { psychiatric ward, } \\
\text { during a year of } \\
\text { systematic clinical } \\
\text { supervision }\end{array}$ & Sweden & $39.7(7.1)$ & $13.5(8.3)$ & SOC-29 & $\begin{array}{c}\text { T1: } 146.6 \\
(21.2) \\
\text { T2: } 153.6 \\
(18.3)\end{array}$ & Pre-post-test design & $\begin{array}{c}\text { Creative Climate } \\
\text { questionnaire (CCQ) } \\
\text { Work-related strain } \\
\text { inventory (WRSI) } \\
\text { Satisfaction with } \\
\text { nursing care and } \\
\text { work (SNCW) }\end{array}$ & $\begin{array}{l}\text { Not significant Improvement in } \\
\text { SOC scores at T1 and T2 } \\
\text { Inverse correlation between SOC } \\
\text { and WRSI }(\mathrm{r}=-0.48, p<0.05) \\
\text { Inverse correlation between SOC } \\
\text { and SNCW, factor involvement }(\mathrm{r}= \\
-0.46, p<0.05)\end{array}$ \\
\hline $\begin{array}{l}\text { Shiu et al., } \\
1998\end{array}$ & $\begin{array}{cc} & \mathrm{I} \\
20 \text { nurses } & \mathrm{f} \\
\text { (all females) } & \mathrm{o} \\
& \mathrm{a}\end{array}$ & $\begin{array}{l}\text { Public health nurses } \\
\text { (females, } \geq 1 \text { children, } \\
\text { promoted to nursing } \\
\text { officer or in charge of } \\
\text { a center, unit or team) } \\
\text { Work perturbed by } \\
\text { interruption signals }\end{array}$ & Hong Kong & 39 & - & SOC-29 & $\begin{array}{l}135.75 \\
(12.27)\end{array}$ & $\begin{array}{l}\text { Cross-sectional } \\
\text { study }\end{array}$ & $\begin{array}{c}\text { Experience sampling } \\
\text { diary (ESD) }\end{array}$ & $\begin{array}{c}\text { Positive correlation between SOC } \\
\text { and perceived goal progress and } \\
\text { perceived control } \\
\text { Low SOC group (scores }<136) \text { has a } \\
\text { lower positive affect }[\mathrm{t}(323)=-6.79, \\
\mathrm{p}<0.001] \text { and higher negative affect } \\
{[\mathrm{t}(321.98)=2.88, \mathrm{p}<0.005] \text {, than the }} \\
\text { high } \mathrm{SOC} \text { group }(\geq 136) \text { in response } \\
\text { to interruption signals. }\end{array}$ \\
\hline
\end{tabular}


Table 1. Cont.

\begin{tabular}{|c|c|c|c|c|c|c|c|c|c|c|}
\hline Author & Sample-Size/sex & Sample & Country & $\begin{array}{l}\text { Mean Age } \\
\text { (SD) }\end{array}$ & $\begin{array}{l}\text { Mean } \\
\text { Experience } \\
\text { (SD) }\end{array}$ & SOC Scale & $\begin{array}{l}\text { Mean SOC } \\
\text { (SD) }\end{array}$ & Study Design & $\begin{array}{l}\text { Other Used } \\
\text { Instruments }\end{array}$ & $\begin{array}{l}\text { Sense of Coherence } \\
\text { (SOC)-Related Results }\end{array}$ \\
\hline $\begin{array}{l}\text { Pålsson et al., } \\
\quad 1996\end{array}$ & $\begin{array}{c}33 \text { nurses } \\
\text { (all females) } \\
\end{array}$ & $\begin{array}{l}\text { District nurses } \\
\text { working in } 10 \text { primary } \\
\text { health care districts } \\
\text { Supervisory group } \\
\text { ( } \mathrm{n}=21) \text { : participation } \\
\text { in a training program } \\
\text { Comparison group }(n \\
=12)\end{array}$ & Sweden & $\begin{array}{c}\text { Supervisory } \\
\text { group: } 49.0 \\
(7.1) \\
\text { Comparison } \\
\text { group: } 46.3 \\
\quad(8.3)\end{array}$ & $\begin{array}{c}\text { Supervisory } \\
\text { group } \\
\text {-in nursing } \\
\text { care } 24.0 \\
(8.2) \\
\text {-as district } \\
\text { nurse } 16.1 \\
(6.5) \\
\text { Comparison } \\
\text { group } \\
\text {-in nursing } \\
\text { care 21.8 } \\
(6.9) \\
\text {-as district } \\
\text { nurse 14.0 } \\
(7.3)\end{array}$ & SOC-29 & $\begin{array}{l}\text { Supervisory } \\
\text { group } \\
\text { T1: } 148(17.5) \\
\text { T2: } 151 \text { (16.6) } \\
\text { Comparison } \\
\text { group } \\
\text { T1: } 154 \text { (13.6) } \\
\text { T2: } 153 \\
\text { (17.3) }\end{array}$ & $\begin{array}{l}\text { Pre-post test design, } \\
\text { with control }\end{array}$ & $\begin{array}{l}\text { Karolinska Scales of } \\
\text { Personality (KSP) } \\
\text { Burnout Measure } \\
\text { Empathy Construct } \\
\text { Rating Scale (ECRS) }\end{array}$ & $\begin{array}{r}\text { Burnout, empathy and SOC scores } \\
\text { are concordant in respect to the KSP } \\
\text { variables } \\
\text { Inverse correlation between SOC } \\
\text { and KSP variables: somatic anxiety } \\
(\mathrm{r}=-0.44, p<0.05) \text {, impulsiveness } \\
(\mathrm{r}=-0.40, p<0.05), \text { monotony } \\
\text { avoidance }(\mathrm{r}=-0.42, p<0.05), \\
\text { detachment }(\mathrm{r}=-0.44, p<0.05), \\
\text { hostility }(\mathrm{r}=-0.37, p<0.05) \text { and } \\
\text { psychasthenia }(\mathrm{r}=-0.40, \mathrm{p}<0.05) \\
\text { Positive correlation between SOC } \\
\text { and KSP variable, socialization }(\mathrm{r}= \\
0.44, p<0.01) \\
\text { Inverse correlation between SOC } \\
\text { and burnout }(\mathrm{r}=-0.69, p<0.001) \\
\text { Positive correlation between SOC } \\
\text { and empathy ( } \mathrm{r}=0.76, p<0.001) \\
\text { No significant change in burnout, } \\
\text { empathy and SOC over time within } \\
\text { the groups nor between the groups } \\
\text { at T1 or at T2 }\end{array}$ \\
\hline $\begin{array}{l}\text { Lewis } \\
1994\end{array}$ & $\begin{array}{l}49 \text { nurses } \\
\text { (all female) }\end{array}$ & $\begin{array}{l}\text { Nurses working in } \\
\text { dialyses units }\end{array}$ & USA & 39.6 & - & SOC-29 & 148.7 & $\begin{array}{l}\text { Cross-sectional } \\
\text { study }\end{array}$ & $\begin{array}{l}\text { Perceived Stress Scale } \\
\text { Nursing Stress Scale } \\
\text { Coping Resources } \\
\text { Inventory } \\
\text { Myers-Briggs Type } \\
\text { Indicator (MBTI) } \\
\text { Maslach burnout } \\
\text { inventory (MBI) }\end{array}$ & $\begin{array}{c}\text { Inverse correlation between SOC } \\
\text { and personal stress }(\mathrm{r}=-0.715) \\
\text { Inverse correlation between SOC } \\
\text { and work stress }(\mathrm{r}=-0.436) \\
\text { Inverse correlation between SOC } \\
\text { and burnout }(\mathrm{r}=-0.395) \\
\text { Positive correlation between SOC } \\
\text { and total coping resources }(\mathrm{r}= \\
0.667)\end{array}$ \\
\hline
\end{tabular}


Table 1. Cont.

\begin{tabular}{|c|c|c|c|c|c|c|c|c|c|c|}
\hline Author & Sample-Size/sex & Sample & Country & $\begin{array}{l}\text { Mean Age } \\
\text { (SD) }\end{array}$ & $\begin{array}{l}\text { Mean } \\
\text { Experience } \\
\text { (SD) }\end{array}$ & SOC Scale & $\begin{array}{l}\text { Mean SOC } \\
\text { (SD) }\end{array}$ & Study Design & $\begin{array}{l}\text { Other Used } \\
\text { Instruments }\end{array}$ & $\begin{array}{l}\text { Sense of Coherence } \\
\text { (SOC)-Related Results }\end{array}$ \\
\hline $\begin{array}{l}\text { Langius et al., } \\
\quad 1992\end{array}$ & $\begin{array}{l} \\
57 \text { nurses } \\
\text { (all females) } \\
\\
\end{array}$ & $\begin{array}{l}\text { Nurses participating } \\
\text { in an in-house } \\
\text { training program } \\
\text { (total } 5 \text { groups) } \\
\text { Sample } 1(n=35): \\
\text { SOC through VAS } \\
\text { format } \\
\text { Sample } 2(n=22) \text { : } \\
\text { SOC and SMI through } \\
\text { VAS format }\end{array}$ & Sweden & $\begin{array}{l}\text { Sample 1: } 41 \\
\text { Sample 2: } 43\end{array}$ & - & SOC-29 & $\begin{array}{c}\text { Sample 1: } \\
\text { 152 (17) } \\
\text { Sample 2: } \\
143(17)\end{array}$ & $\begin{array}{l}\text { Cross-sectional } \\
\text { study }\end{array}$ & $\begin{array}{l}\text { Self-Motivation } \\
\text { Inventory (SMI) }\end{array}$ & $\begin{array}{c}\text { No differences in SOC scores means } \\
\text { between the participating groups, } \\
\text { who had questionnaire } \\
\text { administered through different } \\
\text { formats. } \\
\text { Negative correlation between SOC } \\
\text { and SMI }(\mathrm{r}=-0,685, \mathrm{p}<0.001)\end{array}$ \\
\hline $\begin{array}{l}\text { Lewis } \\
1991\end{array}$ & $\begin{array}{c}238 \text { nurses } \\
224 \text { females } \\
(94 \%) \\
14 \text { males } \\
(6 \%)\end{array}$ & $\begin{array}{l}\text { Nurses working in } \\
\text { dialysis units }\end{array}$ & USA & 36.1 & - & SOC-29 & 143.1 & $\begin{array}{l}\text { Cross-sectional } \\
\text { study }\end{array}$ & $\begin{array}{l}\text { Nursing stress scale } \\
\text { Maslach Burnout } \\
\text { inventory (MBI) }\end{array}$ & $\begin{array}{c}\text { Men show lower SOC scores than } \\
\text { women } \\
\text { Inverse correlation between SOC } \\
\text { and overall stress }(\mathrm{r}=-0.39) \\
\text { Correlation between SOC and } \\
\text { burnout subscales (emotional } \\
\text { exhaustion } \mathrm{r}=-0.57, \\
\text { depersonalization } \mathrm{r}=-0.54, \\
\text { personal accomplishment } \mathrm{r}=0.53) \\
\text { No relationship between SOC and } \\
\text { age, marital status, educational } \\
\text { level, position, years in nursing, } \\
\text { number of patients or shift unit, } \\
\text { shift length or hours worked per } \\
\text { week. }\end{array}$ \\
\hline
\end{tabular}




\subsection{Work-Related Variables}

\subsubsection{Job Characteristics}

Debska et al. observed among the nurses highest SOC scores for the Manageability subscale (45.15), followed by the Comprehensibility and Meaningfulness subscales. They showed an inverse correlation between SOC and the dimensions of mental load investigated by the Meister questionnaire, such as Monotony, Unspecific Load and Mental Load [6]. The relationship between SOC and general working experience, position at work and employment characteristics was unclear, while some authors found no correlation $[7,8]$, an inverse correlation between SOC and work experience was found by Debska et al. [6], in contrast Miyata et al. [9] observed a positive correlation. Among nurses there is a wide variety of work schedule such as regular, irregular, flexible, etc. Fusz et al. showed that day-shift workers had higher SOC score than shift workers, and that lower SOC value was found among irregular workers (58.19), compared to flexible work schedule workers [10], while Kikuchi et al. observed an inverse correlation between SOC and shift work, job rank, and overtime hours [11]. Several studies found differences of SOC between different professionals, because there was higher SOC in nurses employed as strategic managers [7], Lindmark et al. likewise showed that clinical coordinators have higher SOC score, compared with all other professions, for example dental hygienists have higher scores for meaningfulness, and dentists have higher scores for manageability, compared with dental nurses [12].

Ando et al. described the relationship between the moral distress for nurses and several job characteristics, such as job satisfaction, SOC and mental health, finding an inverse correlation between Moral Distress Scale for Psychiatric Nurses (MDS-P) and SOC. Inverse correlations were found between subscales of the MDS-P and those of SOC [13]. Positive correlation was observed between SOC and workplace adaptability [14], and job satisfaction [11,14,15]. Moreover, Ida et al. identified SOC as an important factor affecting sickness-absence [14].

Lastly planning effective pain and distress management is a crucial part of the nurses' profession. Hall-lard et al. found that patient's age and type of illness seems to influence nurses' assessments of pain and distress, nurses with high emotional stability and high SOC scores assess pain and distress for acute patients as less intense and assess it more intense for chronic patients [16].

\subsubsection{Work-Life Balance}

Some authors, investigating Work-Life Balance as the proportions of percentages of time spent at work and private life (50/50 and below, 60/40, 70/30, 80/20 and above), reported significantly higher SOC scores in "50/50 and below" and "60/40" groups, whereas the lowest SOC scores were associated with the "80/20 and above" group [2,17].

As far as the Quality of Life (QoL) is concerned, the "50/50 and below" reported the higher scores for overall QoL and physical health, while the "80/20 and above" group the lowest in the overall QoL, in the physical health domain and in the environment domain. No significant differences among the four groups were observed in terms of social support, job satisfaction, and the psychological and social relationship domains of the QoL [2].

\subsubsection{Work Related Trauma}

Michael et al. investigated the effect of social and personal resources at work, related to trauma. They observed that nurses who did not report a traumatic event had the strongest SOC. This could be due to some causes, nurses with strong SOC did not perceive an event as traumatic, or in contrast, traumatic events influence the SOC [18].

\subsubsection{Social Support}

Social support and SOC were found to be significant predictors $(p<0.05)$ for all QoL domains. Indeed, a unit increase in SOC results in a 6-12\% increase in the likelihood of having high QoL for all 
domains, however social support had more influence on nurses' QoL than their ability to cope with stress [2].

\subsubsection{Stress and Burnout}

Yam et al. analyzed SOC and perceived stress with a sample of critical care nurses, finding that SOC was a protective factor in relation to stress perceptions arising from the work environment [19].

Höge et al. investigated the possible impact of SOC and negative affectivity on the relationship between work stressors and strain. They found a strong correlation between SOC and negative affectivity [20].

Berg et al. [21] observed that Work-Related Strain Inventory (WRSI), measuring the feeling of psychological strain in occupational setting, and factor involvement of the Satisfaction with Nursing Care and Work (SNCW) scale, negatively related to SOC.

Several studies negatively correlated SOC with overall stress [8,20] and work-related stress [8], especially workload [22]; in these studies, nurses' overload in the workplace was identified as a critical factor for stress development. Burnout and SOC were found to inversely correlate in several studies [22-25].

Moreover, burnout subscales were observed to logically relate to SOC. A stronger coping ability is associated with higher scores in personal accomplishment, lower levels of emotional exhaustion, and depersonalization $[8,15,26,27]$. Workload was considered a major contributing factor for burnout [8].

\subsection{Individual Variables}

\subsubsection{Individual Characteristics}

Five studies investigated the correlation between SOC and age, with discordant results: two studies $[11,28]$ revealed a positive association between SOC and age of the participants, whereas another three studies $[7,8,29]$ did not find this relationship significant. Nevertheless, in the study conducted by Debska et al., although no significant correlation was found between total SOC and age, an inverse association between age and Manageability subscale was observed [6].

Although one study did not find any correlation with sex of the participants [26], an earlier study by Lewis [8] observed a stronger SOC in women, compared to men.

SOC was associated to marital status in the study conducted by Tselebis et al. [26], whereas the same correlation was not found in other studies [8,9].

Educational background, considered by Kretowicz et al. was found to be positively associated to overall SOC and Meaningfulness [7]. Two studies by Debska et al. and Lewis et al. have not proven the same correlation $[6,8]$.

\subsubsection{Individual Physical and Mental Health}

The relationship between SOC and nurses' health was the focus of several studies. Miyata et at. associated positively SOC with good mental health status and good physical health status [9].

Schäfer et al. observed a significant increase from the cut-off value of nurses' scores in ICD-10-Symptoms Rating (ISR), evaluating general health problems, as well as symptoms burden, depression and eating disorder symptoms. Moreover, when compared to physicians, nurses reported higher ISR and symptoms burden scores, the same was not found for variables such as Resilience, SOC or LOC (Locus of Control). Furthermore, SOC, Resilience, and Internal and External LOC correlated with ISR scores and Post-Traumatic Stress Disorder (PTSD) symptoms, correlating SOC as a significant predictor of mental health problems and of symptom severity [30].

An inverse correlation was found between health risk and SOC, the latter significantly affecting sickness-absences, especially for experienced and expert nurses, for whom it is the only casual factor, among the other investigated variables [14]. 
Depression and SOC have been found to negatively, and strongly, correlate in several studies [11, $26,28,31]$. Takeuchi et al. also considered the interaction of SOC and work-family conflicts (WFC) on the degree of nurses' depression and pointed out the buffering effect of SOC against depression, resulting from WFC [31].

Moreover, an inverse correlation was found between SOC and personal stress [22] and cumulative fatigue [31].

\subsubsection{Personality Traits and Characteristics}

Van der Colff et al. showed that SOC was correlated positively with different coping strategies, evaluated through the Coping Orientation for Problem Experienced (COPE) questionnaire, namely Approach Coping (seeking emotional/social support) and Turning to Religion; the correlation was inverse for Avoidance and Focus on and ventilation of emotions [15].

Overall a higher SOC score was associated with stronger total coping resources [22], thriving and the use of GRRs [1] and greater self-motivation, measured by the Self-Motivation Inventory (SMI) [32].

SOC was positively related to perceived progress goal as well as perceived control, both related to the perception of characteristic tasks of the job and life activities in which nurses were involved, evaluated upon interruption signals. Such signals were found to have a lower positive affect and higher negative affect in nurses with lower SOC [33].

Few studies concentrated on the relationship between SOC and personality traits. Kikuchi et al. revealed that SOC had a strong correlation with almost all personality traits, the strongest being the one with Neuroticism [11]. Höge et al. underlined the same concordant relationship between SOC and Negative Affectivity [20]. Similarly, SOC was found to correlate to the Karolinska Scale of Personality (KSP): negatively to Impulsiveness, Monotony Avoidance, Detachment, Hostility and Psychasthenia, and positively to Socialization and Empathy [23].

The KSP variable "Somatic Anxiety" was inversely related to SOC [23], but in contrast, no differences in mean SOC between the Anxiety (+) and the Anxiety (-) groups were found by Yoshida et al. [34].

\subsubsection{Negative Life Events}

In two different studies Hochwälder et al. investigated the association of negative life events on nurses' SOC $[29,35]$. There was no strong evidence that negative life events lower SOC in the sample population, but those who experienced a negative life event had initially a weaker SOC, compared to those who did not experience any negative life events [35]. Although there was not a significant correlation between SOC and the number of uncontrolled negative life events, those with high and moderate SOC reported fewer controllable negative life events compared to individuals with low SOC [29].

\subsection{Intervention Studies}

A total of six studies analyzed the effect of an intervention on nurses' Sense of Coherence. Only two studies observed a significant improvement in the SOC scores [36,37]. In the first study, nurses participated in a modified version of the Mindfulness-Based Stress Reduction (MBSR) program, lasting two weeks. After the intervention, it was observed a significant decrease in GHQ and its subscales (Physical Symptoms, Anxiety/Sleep, Social Activities and Depression), indicating an overall improvement in general health. Furthermore, SOC increase was significant, compared to the control group, as it was the increase in the meaningfulness subscale score, compared to comprehensibility and manageability scores [36].

Sarid et al. investigated the effect of Cognitive-Behavioral Intervention (CBI), comprised of 16 meetings, once a week, on nurses' SOC. At baseline the two groups did not differ in respect to SOC, perceived stress and mood states $[37,38]$. At T2 (four months after the beginning, upon completion of 
the program), nurses of the intervention group scored higher in SOC and vigor scales, whereas reported decreased level of perceived stress and fatigue. Such changes were not reported in the control group.

Nurses in the study conducted by Shimizu participated in an Assertive Training program. Although no significant changes in SOC were reported, the effects of the intervention were appreciable as an improvement in Self-esteem scores in the sample analyzed [39].

Berg 1999 and Pålsson 1996 both investigated the outcomes of systematic clinical supervision strategies on nurses. The two studies did not report significant changes in SOC after the intervention [21,23].

Only one study observed a reduction of mean SOC scores of nurse managers in early years of their supervisory roles, after the participation in a four-month experiential learning-based program [40].

The effect of an IT support project on SOC was considered in one study: no significant within-subject effects for the total SOC scale and meaningfulness subscale was observed both in the group receiving the intervention and the control group. However, IT support improved the perception of psychosocial job satisfaction and the quality of care; in this sense the study showed a significant interaction effect for the family relation factors, close friend relation (LSQ), total SOC scale and meaningfulness subscale [41].

\section{Discussion}

The nursing profession is characterized by taking care of patients and their families, it is a factor increasing the mental and emotional burden, and for this reason nurses' Sense of Coherence needs to be strong enough to deal with several stressful working experiences. Among others, most of the strain experienced by nurses derives from heavy workload, unsatisfactory work environment and work conditions, deep emotional involvement in others, organizational structure, lack of resources, inter-professional conflicts and professional uncertainty [21].

The majority of nurses spend more time at work than on their private life and report significantly higher SOC scores for those whose percentages were proportionally lower, and the lowest scores were for nurses with higher percentages of time spent on working activities [2,17].

Nurses face moral distress and feel so powerless because of the management policy of institutions [13]. The crucial role of institutions in cooperating in the hospital management is also correlated to a positive perception of safety, which in turn is correlated with absence of burnout and a strong Sense of Coherence [24].

The raising of SOC and organization environment reduces sickness-absence. Improving comprehensibility by enriching professionalism, recovering meaningfulness and manageability through optimizing work-life balance and social support may also raise SOC.

SOC and social support were found to be significant predictors for all QoL domains. Social support had the most relevant influence on nurses' QoL and is considered as a buffer in the stressful situations of healthcare working environment to help the individuals to cope. Cultivating social support could indeed help the individuals to improve their coping abilities and their general health status [2].

Occupational stress is a major contributing factor to burnout [15]. This correlation is also supported by studies, showing that individuals with high burnout levels are expected to possess poor stress coping abilities, specifically in the manageability dimension of the Sense of Coherence, which was found to be related to emotional exhaustion of burnout [15]. Burnout is defined as a syndrome of emotional exhaustion, depersonalization and decreased sense of self-achievement, unfortunately, occupational burnout affects a considerable proportion of nurses who face daily stress experienced at work [25].

Despite the relative stability of SOC after the third decade, it may be shaped progressively throughout the whole course of someone's life and the GRRs [42], which are mobilized by the Sense of Coherence, arise from the cultural, social and environmental conditions of living, in addition to idiosyncratic factors [43]. 
This concept could explain why the analysis of the correlation between SOC and individual characteristics (age, sex, marital status and educational background), taken into account only in few studies, yield discordant results.

An interesting point was explored by Kretowicz et al., who correlated positively SOC and educational background: as SOC is considered to have an educational value and the progress in the academic education could elevate it, it is reasonable to think that this relationship could influence task completion in managerial positions [7].

Furthermore, Antonovsky did not exclude a possible influence of negative life events on SOC, especially for those with low or moderate SOC [42]. Starting from this assumption, Hochwälder et al. in two different studies $[29,35]$ have investigated this relationship: no association was found between negative life events and nurses' SOC, however those who experienced a negative life event had initially a weaker SOC, compared to those who did not experience any [35]. This result led the authors to consider a low SOC as a vulnerability factor, rather than considering a high SOC as a protective factor. This finding is in accordance with Antonovsky's assumption that a high SOC could prevent the experience of negative life events, both helping individuals to avoid potential stressors and not allowing them to perceive them invariably as negative [4].

A strong SOC is believed to be related to general well-being [15]. This relationship was confirmed by Miyata et al., who demonstrated in nurses a positive association between SOC and good mental health status and good physical health status [9].

Moreover, nurses working in hospitals reported, compared to the general population, higher burden of general health problems, as well as symptoms of burden, depression and eating disorders symptoms. SOC was found to be the most important predictor for general mental health problems and post-traumatic stress symptoms. SOC could play a crucial role in the development and course of these health issues, by shaping the perception and attitude toward aversive work experiences and stress [30].

The inverse relationship between SOC and health risk and the identification of SOC as a key determinant of sickness absences demonstrates how a poor coping ability, in the presence of powerful stressors, such as advanced career levels, could represent a health risk, due to a decreased ability to cope successfully with the stress. Nevertheless, Ida et al. advanced the possibility that raising SOC and the organization of the environment could produce a positive effect on sickness absences [14].

The inverse correlation between depression and SOC was strong in several studies [26,30,31].

Possession of a strong SOC allowed nurses to better manage occupational stress due to lack of organizational support and job demands, through the choice of appropriate coping strategies [15], to define themselves as thriving, with a positive use of GRRs [1] and to possess a greater self-motivation [32]. Self- motivation was found related to certain specific behavior attitudes (propensity toward physical activity and giving it value in respect to health) and the hypothesis of Langius et al. of a positive relation to SOC was confirmed by their investigation [32].

Only two studies investigated the correlation between anxiety and SOC. Yoshida et al. confronted two groups, divided based on anxiety presence, assessed by an ad hoc questionnaire: no differences were found among the two groups, possibly explained by the initially high SOC possessed by the group at hand [34]. Palsson et al.'s finding indicated that there is an inverse relationship between self-rated pathogenic anxiety and self-rated salutogenic Sense of Coherence [23].

Among the five studies investigating the effects of an intervention on the SOC, only two studies reported significant results. Stress coping strategies improvement, achieved by the MBSR therapy was demonstrated by a significant increase in SOC scores. Moreover, a significant increase in the Meaningfulness subscale of SOC indicated that, through the program, nurses were able to focus their attention on mind and body, allowing them to find meaning in their life and work activities [36].

The effects of CBI were significant in increasing SOC and vigor levels and in decreasing perceived stress and fatigue. CBI aims to raise the personal awareness on possible stress reaction, to learn how to self-talk in anxiety-producing situation, to gain a balance and awareness on perspective stressful events 
and to facilitate cognitive restructuring of stressful work situations. These intrinsic characteristics of the therapy explain the improvement in nurses' coping ability and the reduction of negative moods $[37,38]$.

The only study reporting a decrease in mean SOC score investigated the changes produced by an experiential learning-based program. This result was explained by the overload experienced by nurse managers in early years of their supervisory roles when discussing their behavior and stressful situation encountered at work. Furthermore, it has been argued that SOC could possibly increase after an initial decrease, which was not evaluated, due to the short-term follow-up performed [40].

The other intervention studies did no show significant changes on SOC, these results are coherent with the initial description of SOC by Antonovsky, for whom SOC tends to remain stable in adulthood under normal circumstances and can be considered as a moderating factor on negative work environment variables [21]. Moreover, also the well-recognized difficulty in obtaining a significant SOC change in respect to high or low baseline is considered a determining factor of SOC stability in these studies [23].

\section{Conclusions}

SOC provides a solid theoretical basis for examining the organization of work [32].

Therefore it has been proposed that nursing management could focus on building a healthy work environment, which fosters SOC, rather than concentrating on resolving the effects of stress and its management at individual level [33].

Comprehensibility is improved by a clear view on roles and responsibilities and by open communication channels; sense of manageability is strengthened by appropriate workload and availability of resources [8,22]. Participation in decision making and the perspective of a clear career path are factors improving the sense of meaningfulness [8,33].

We found that SOC was a protective factor for depressive state, burnout, job dissatisfaction among female nurses, but there is no clear correlation with factors such as working experience or position at work. In addition, a higher SOC enhances a good mental and physical health status, acting as a health promoting resource, according to Antonovsky's theory [44,45].

Author Contributions: Conceptualization, G.M.M. and M.C.; Methodology, M.C. and S.P.; Formal Analysis, G.M.M. and M.C.; Investigation, E.A. and C.S.; Resources, M.C. and E.A.; Data Curation, S.P.; Writing-Original Draft Preparation, M.C.; Writing—Review \& Editing, G.M.M. and M.C.; Visualization, G.M.M.; Supervision, G.M.M.; Project Administration, G.M.M. All authors have read and agreed to the published version of the manuscript.

Funding: This research received no external funding.

Conflicts of Interest: The authors declare no conflict of interest.

\section{References}

1. Stock, E. Exploring salutogenesis as a concept of health and wellbeing in nurses who thrive professionally. Br. J. Nurs. 2017, 26, 238-241. [CrossRef]

2. Kowitlawkul, Y.; Yap, S.F.; Makabe, S.; Chan, S.; Takagai, J.; Tam WW, S.; Nurumal, M.S. Investigating nurses' quality of life and work-life balance statuses in Singapore. Int. Nurs. Rev. 2018, 66, 61-69. [CrossRef]

3. Ilić, I.M.; Arandjelović, M.Ž.; Jovanović, J.M.; Nešić, M.M. Relationships of Work-Related Psychosocial Risks, Stress, Individual Factors and Burnout—Questionnaire survey among emergency physicians and nurses. Med. Pr. J. 2017, 68, 167-178. [CrossRef]

4. Antonovky, A. Health, Stress, and Coping; Jossey-Bass: San Francisco, CA, USA, 1979.

5. Mittelmark, M.B.; Sagy, S.; Eriksson, M.; Bauer, G.F.; Pelikan, J.M.; Lindström, B.; Espnes, G.A. The Handbook of Salutogenesis; Springer: Berlin, Germany, 2017.

6. Hobfoll, S.E. Conservation of Resources: A New Attempt at Conceptualizing Stress. Am. Psychol. 1989, 44, 513-524. [CrossRef]

7. Dębska, G.; Pasek, M.; Wilczek-Rużyczka, E. Sense of coherence vs. mental load in nurses working at a chemotherapy ward. Cent. Eur. J. Public Health 2017, 25, 35-40. [CrossRef] 
8. Kretowicz, K.; Bieniaszewski, L. Determinants of sense of coherence among managerial nursing staff. Ann. Agric. Environ. Med. 2015, 22, 713-717. [CrossRef]

9. Lewis, S.L.; Campbell, M.A.; Becktell, P.J.; Cooper, C.L.; Bonner, P.N.; Hunt, W.C. Work stress, burnout, and sense of coherence among dialysis nurses. ANNA J. 1992, 19, 545-554.

10. Miyata, C.; Arai, H.; Suga, S. Characteristics of the nurse manager's recognition behavior and its relation to sense of coherence of staff nurses in Japan. Collegian 2015, 22, 9-17. [CrossRef] [PubMed]

11. Fusz, K.; Tóth, Á.; Varga, B.; Rozmann, N.; Oláh, A.; Tudományegyetem, P. Different work schedules of nurses in Hungary and their effects on health. Ideggyógyászati Szle. Clin. Neurosci. 2017, 70, 136-139. [CrossRef] [PubMed]

12. Kikuchi, Y.; Nakaya, M.; Ikeda, M.; Okuzumi, S.; Takeda, M.; Nishi, M. Sense of Coherence and Personality Traits Related to Depressive State. Psychiatry J. 2014, 2014, 738923. [CrossRef] [PubMed]

13. Lindmark, U.; Wagman, P.; Rolander, B. Workplace health in dental care-A salutogenic approach. Int. J. Dent. Hyg. 2018, 16, 103-113. [CrossRef] [PubMed]

14. Ando, M.; Kawano, M. Relationships among moral distress, sense of coherence, and job satisfaction. Nurs. Ethics 2018, 25, 571-579. [CrossRef] [PubMed]

15. Ida, H.; Miura, M.; Komoda, M.; Yakura, N.; Mano, T.; Hamaguchi, T.; Yamazaki, Y.; Kato, K.; Yamauchi, K. Relationship between stress and performance in a Japanese nursing organization. Int. J. Health Care Qual. Assur. 2009, 22, 642-657. [CrossRef] [PubMed]

16. Van der Colff, J.J.; Rothmann, S. Occupational stress, sense of coherence, coping, burnout and work engagement of registered nurses in South Africa. SA J. Ind. Psychol. 2009, 35, 1-10. [CrossRef]

17. Hall-lord, M.L.; Larsson, B.W. Registered nurses' and student nurses' assessment of pain and distress related to specific patient and nurse characteristics. Nurse Educ. Today 2006, 26, 377-387. [CrossRef]

18. Makabe, S.; Takagai, J.; Asanuma, Y.; Ohtomo, K.; Kimura, Y. Impact of work-life imbalance on job satisfaction and quality of life among hospital nurses in Japan. Ind. Health 2015, 53, 152-159. [CrossRef]

19. Michael, R.; Jenkins, H.J. Recovery from work-related trauma by perioperative nurses. Collegian 2001, 8, 8-13. [CrossRef]

20. Yam, B.M.C.; Shiu, A.T. Perceived stress and sense ofcoherence among critical care nurses in Hong Kong: A pilot study. J. Clin. Nurs. 2003, 12, 144-146. [CrossRef]

21. Höge, T.; Büssing, A. The Impact of Sense of Coherence and Negative Affectivity on the Work Stressor-Strain Relationship. J. Occup. Health Psychol. 2004, 9, 195-205. Available online: http://doi.apa.org/getdoi.cfm?doi= 10.1037/1076-8998.9.3.195 (accessed on 10 February 2020). [CrossRef]

22. Berg, A.; Hallberg, I.R. Effects of systematic clinical supervision on psychiatric nurses' sense of coherence, creativity, work-related strain, job satisfaction and view of the effects from clinical supervision: A pre-post test design. J. Psychiatr. Ment. Health Nurs. 1999, 6, 371-381. [CrossRef]

23. Lewis, S.L.; Bonner, P.N.; Campbell, M.A.; Cooper, C.L.; Willard, A. Personality, stress, coping, and sense of coherence among nephrology nurses in dialysis settings. ANNA J. 1994, 21, 325-335; discussion 336. [PubMed]

24. Palsson, M.; Norberg, A.; Bjorvell, H. Burnout, Empathy and Sense of Coherence among Swedish District Nurses before and after Systematic Clinical Supervision. Scand. J. Caring Sci. 1996, 10, 19-26. [CrossRef] [PubMed]

25. Vifladt, A.; Simonsen, B.O.; Lydersen, S.; Farup, P.G. The association between patient safety culture and burnout and sense of coherence: A cross-sectional study in restructured and not restructured intensive care units. Intensive Crit. Care Nurs. 2016, 36, 26-34. [CrossRef] [PubMed]

26. Basinska, M.; Andruszkiewicz, A.; Grabowska, M. Nurses' sense of coherence and their work related patterns of behaviour. Int. J. Occup. Med. Environ. Health 2011, 24, 256-266. [CrossRef]

27. Tselebis, A.; Moulou, A.; Ilias, I. Burnout versus depression and sense of coherence: Study of Greek nursing staff. Nurs. Health Sci. 2001, 3, 69-71. [CrossRef]

28. Cilliers, F. Burnout and salutogenic functioning of nurses. Curationis 2003, 26, 62-74. [CrossRef]

29. Kikuchi, Y.; Nakaya, M.; Ikeda, M.; Okuzumi, S.; Takeda, M.; Nishi, M. Relationship between depressive state, job stress, and sense of coherence among female nurses. Indian J. Occup. Environ. Med. 2014, 18, $32-35$. [CrossRef]

30. Hochwalder, J. Test of antonovsky's postulate: High sense of coherence helps people avoid negative life events 1. Psychol. Rep. 2015, 116, 363-376. [CrossRef] 
31. Schäfer, S.K.; Lass-hennemann, J.; Groesdonk, H.; Volk, T.; Michael, T. Mental Health in Anesthesiology and ICU Staff: Sense of Coherence Matters. Front. Psychiatry 2018, 9, 440. [CrossRef]

32. Takeuchi, T.; Yamazaki, Y. Relationship between work-Family conflict and a sense of coherence among Japanese registered nurses. Jpn. J. Nurs. Sci. 2010, 7, 158-168. [CrossRef]

33. Langius, A.; Bjorvell, H.; Antonovsky, A. The Sense of Coherence Concept and its Relation to Personality Traits in Swedish Samples. Scand. J. Caring Sci. 1992, 6, 165-171. [CrossRef] [PubMed]

34. Shiu, A.T.Y. The Significance of Sense of Coherence for the Perceptions of Task Characteristics and Stress During Interruptions Amongst a Sample of Public Health Nurses in Hong Kong: Implications for Nursing Management. Public Health Nurs. 1998, 15, 273-280. [CrossRef] [PubMed]

35. Yoshida, K.; Orita, M.; Goto, A.; Kumagai, A.; Yasui, K.; Ohtsuru, A.; Hayashida, N.; Kudo, T.; Yamashita, S.; Takamura, N. Radiation-related anxiety among public health nurses in the Fukushima Prefecture after the accident at the Fukushima Daiichi Nuclear Power Station: A cross-sectional study. BMJ Open 2016, 6, e013564. [CrossRef] [PubMed]

36. Hochwa“lder, J.; Forsell, Y. Is Sense of Coherence Lowered by Negative Life Events ? J. Happiness Stud. 2011, 12, 475-492. [CrossRef]

37. Ando, M.; Natsume, T.; Kukihara, H.; Shibata, H.; Ito, S. Efficacy of mindfulness-based meditation therapy on the sense of coherence and mental health of nurses. Health 2011, 3, 118-122. [CrossRef]

38. Sarid, O.; Berger, R.; Segal-engelchin, D. The impact of cognitive behavioral interventions on SOC, perceived stress and mood states of nurses. Procedia. Soc. Behav. Sci. 2010, 2, 928-932. Available online: http: //dx.doi.org/10.1016/j.sbspro.2010.03.128 (accessed on 10 February 2020). [CrossRef]

39. Orly, S.; Rivka, B.; Rivka, E.; Dorit, S. Are cognitive-Behavioral interventions effective in reducing occupational stress among nurses? Appl. Nurs. Res. 2012, 25, 152-157. [CrossRef]

40. Shimizu, T.; Kubota, S.; Mishima, N.; Nagata, S. Relationship between Self-Esteem and Assertiveness Training among Japanese. J. Occup. Health 2004, 46, 296-298. [CrossRef]

41. Kuraoka, Y. Effect of an experiential learning-Based programme to foster competence among nurse managers. J. Nurs. Manag. 2018, 26, 1015-1023. [CrossRef]

42. Engström, M.; Ljunggren, B.; Lindqvist, R.; Carlsson, M. Staff perceptions of job satisfaction and life situation before and 6 and 12 months after increased information technology support in dementia care. J. Telemed. Telecare 2005, 11, 304-309. [CrossRef]

43. Antonovsky, A. Unraveling the Mystery of Health. How People Manage Stress and Stay Well; Jossey-Bass: San Francisco, CA, USA, 1987.

44. Eriksson, M.; Lindstrom, B. Validity of Antonovsky's sense of coherence scale: A systematic review. J. Epidemiol. Community Health 2005, 59, 460-466. [CrossRef] [PubMed]

45. Eriksson, M.; Lindström, B. Antonovsky's sense of coherence scale and the relation with health: A systematic review. J. Epidemiol. Community Health 2006, 60, 376-381. [CrossRef] [PubMed] 